\title{
Internal and External Factors Influencing Millennials' Sharing Behaviour of Online Video Advertisements
}

\author{
Nik Mohamad Shamim Nik Mohd Zainordin ${ }^{1}$, Norazlyn Kamal Basha ${ }^{1}$, Ho Jo Ann ${ }^{1}$ \& Ng Siew Imm ${ }^{1}$ \\ ${ }^{1}$ School of Business and Economics, Universiti Putra Malaysia, Serdang, Selangor, Malaysia \\ Correspondence: Norazlyn Kamal Basha. E-mail: norazlyn@upm.edu.my
}

Received: November 15, 2021

doi:10.5539/ass.v18n1p23
Accepted: December 11, 2021

Online Published: December 30, 2021

URL: https://doi.org/10.5539/ass.v18n1p23

\begin{abstract}
This study aimed to uncover the internal and external factors driving Millennial consumers to engage in Online Video Advertisements (OVAs) sharing. Underpinned by the Theory of Planned Behaviour (TPB), the internal factors tested in this study were attitude, subjective norms, and perceived behavioural control. The external factors predicted to influence consumers' intention and actual sharing behaviour of OVAs were company reputation, brand awareness, and celebrity endorsement were underpinned by Stimulus Organism Response Model (S-O-R). The study included perceived intrusiveness as a moderator between the aforementioned antecedents and the sharing behaviour of OVAs. A total of 220 Millennial respondents was collected in Selangor, Malaysia. Partial Least Squares Structural Equation Modelling (PLS-SEM) analysis showed that attitude and subjective norms significantly predict consumers' intention to share OVAs. In terms of external antecedents, only celebrity endorsements were found to positively influence the sharing intention of OVAs. The study also revealed that perceived intrusiveness negatively moderates the effects of attitude and social norms on the intention to share OVAs.
\end{abstract}

Keywords: Online Video Advertisements (OVAs), Millennial, Theory of Planned Behaviour (TPB), Stimulus Organism Response Model (S-O-R), Partial Least Squares Structural Equation Modelling (PLS-SEM)

\section{Introduction}

The ever-increasing quantity and variety of multimedia content has changed the way people watch and share videos online. Internet users can now watch videos on numerous platforms, from streaming videos on TVs and gaming consoles from different content providers, to downloading videos to their computers or smartphone devices. According to McCue (2020), online videos will exceed $82 \%$ of all internet traffic by the year 2022, which is 15 times higher than it was in 2017. The same report projects that around $49 \%$ of people watch five or more videos per day (McCue, 2020). According to YouTube Press' (2021) official statistics, over two billion users visit YouTube every month; these uses collectively spend billions of hours watching YouTube videos every day. Moreover, as reported by the Malaysian Communications and Multimedia Commission (MCMC, 2021), a massive jump has been seen in the number of hours spent on the internet following the Covid-19 pandemic, driving the proportion of internet users who watch videos online to $87 \%$.

It is not surprising, therefore, that online video advertisements (OVAs) are now one of the most popular tools among marketers. OVAs are useful not only because viewers are able to share these advertisements across various online platforms, but also because $64 \%$ of customers make purchase decisions after seeing a branded video advertisement on a social media platform (Lister, 2018). However, a majority of viewers have been found to skip OVAs; thus, they are not likely to fully view OVAs and are even less likely to share OVAs (Marketing Land, 2016). In fact, users tend to close an advertisement page or skip the advertisement pre-roll, mid-roll, or post-roll (eMarketer, 2016). Notably, the MCMC (2017) reported that most internet users are from the Millennial generation, i.e., aged between 19 to 38 (born at year 1981 to 2000, Kraus, 2017), making them the primary target audience of OVAs. Given that this generation is incredibly sophisticated, technology savvy, and immune to most traditional marketing and sales pitches (Hall, Towers \& Shaw, 2017; Ladhari, Gonthier \& Lajante, 2019), the current research attempted to contribute to the marketing literature and practice by examining the antecedents of OVA sharing behaviour among Millennials in Selangor, Malaysia.

\section{Online Video Advertisements (OVAs)}

OVAs, also known as internet advertising and digital advertising, uses internet technologies to advertise through videos containing information about products or services (Lee et al., 2021). When a company advertises its 
products or services on an online medium at little to no cost, it can reach interested consumers in any part of the world that has access to internet connection. Many marketers thus produce video advertisements specifically for online platforms, as this medium typically receives more favourable responses from consumers. Consistent with this, Pashkevich et al. (2012) stated that advertising through video streams such as YouTube and Facebook is more effective than advertising through traditional mediums. Moreover, if a video advertisement is entertaining and useful to consumers, they are likely to share the video with other people, which creates a 'snowball' advertising effect. For instance, Quesenberry and Coolsen (2019) revealed that OVAs on social media like Facebook and YouTube can go viral when the video spreads rapidly through online sharing and reaches millions of viewers. Further proving that people tend to watch online videos, MCMC's (2021) report showed that YouTube was the second most popular platform among Malaysians after Facebook, and the usage of YouTube was much higher $(80.6 \%)$ in 2020 than in 2018 (48.3\%).

Scholars have recently begun looking into the context of OVAs. For instance, Belanche Flavián and Pérez-Rueda (2017) study investigated the influence of high-arousal and low-arousal stimuli on skippable ad effectiveness (i.e., ad acceptance, ad attitude, brand attitude, and ad intrusiveness). Their results revealed the positive influence of high-arousal stimuli on ad effectiveness. On the other hand, Quesenberry and Coolsen (2019) explored three factors that influence online video virality (i.e., the likelihood of a video being shared and viewed), which are full story development, time duration, and company size. They found a significant effect of full story development on online video virality, but not of time duration and company size on online video virality.

\section{Hypotheses Development}

\subsection{Theoretical Foundation}

The Theory of Planned Behaviour (TPB) was the main theory of this study, based on which the conceptual framework was constructed. The TPB proposes three predictors of behavioural intention: attitude towards the behaviour, i.e., the degree to which an individual has a favourable or unfavourable appraisal of the behaviour in question; subjective norm, i.e., the perceived social pressure to perform or not to perform the behaviour; and perceived behavioural control (PBC), i.e., the perceived ease or difficulty of performing the behaviour which reflects one's experience as well as predicted weaknesses and problems (Ajzen, 1991). The TPB has been widely applied in the advertising context to understand the effects of online advertising on consumers' perceptions and subsequent behaviours. For example, Sanne and Wiese (2018) applied the TPB in understanding and predicting users' engagement with Facebook advertising, while Leung and Chen (2017) employed the TPB to explain the intention of Hong Kong users to accept mobile advertising based on their lifestyle, contextual factors, mobile viewing habits, TV content interest, and intention to adopt mobile television.

In this study, the TPB was selected as the theoretical framework over the Theory of Reasoned Action (TRA) due to the former's inclusion of the PBC factor. Armitage and Conner (2001) revealed that the TPB added the PBC component to incorporate an estimate of behaviours that are not under complete volitional control. Comparing the capabilities of the TRA and the TPB, Hansen et al. (2004) confirmed that the TPB offers the best fit to the data and clarifies the highest amount of variance in online grocery buying intention. This implies that the TPB is more suitable for studies that seek to measure behavioural intention. Therefore, the current study was justified in applying the TPB to explain the internal factors that influence the intention to share OVAs.

The Stimulus-Organism-Response (S-O-R) model of environmental psychology (Mehrabian \& Russell, 1974) advocates that an environmental stimulus ( $\mathrm{S}$ ) triggers the internal state of individuals $(\mathrm{O})$, which in turn, manifests as their behavioural response (i.e., decision to avoid or approach a behaviour) (R). The S-O-R model has been used broadly in consumer behaviour studies, such as consumer impulse buying behaviour in a mobile auction (Chen \& Yao, 2018), consumer online repurchase intention (Zhu, Kowatthanakul \& Satanasavapak, 2019), consumer behaviour towards brand-related user-generated content through Facebook (Arif, Aslam \& Siddiqui, 2020), and the effects of online reviews' information quality and social presence on purchase intention (Zhu et al., 2020). Based on the strong empirical support for this theory, the current study applied the S-O-R model to explain three external factors (company reputation, brand awareness, and celebrity endorsements) that influence the intention to share OVAs.

The current study also adopted the Psychological Reactance Theory (PRT) to support the moderator, perceived intrusiveness. The PRT refers to a certain action that threatens the freedom of people to act or behave as they normally would (Brehm, 1996). This occurs when people respond to an external factor which makes them believe their freedom is limited, wherein their behaviour is regarded as reactance arousal (Baumeister et al., 2002). Edwards, Li and Lee (2002) were the first to apply this theory to identify forced exposure to online advertising. They defined perceived intrusiveness as advertising elements that cause irritation and avoidance by 
interrupting consumers' activities when viewing online content. The PRT has been used to examine the effect of online behavioural advertising on attitude and purchase intention (Fachryto \& Achyar, 2018), as well as to analyse consumers' psychological reactance and ownership in in-game advertising (Malhotra, Mishra \& Saxena, 2021). Notably, Hoogma (2015) applied perceived intrusiveness as moderator between the effect of email marketing on repeat purchase behaviour and brand attitude. Following these findings, in the current study, the PRT was used to justify perceived intrusiveness as a moderator between the TPB factors and the intention to share OVAs.

\subsection{Attitude Towards Sharing OVAs}

Attitude towards a behaviour is defined as individuals' feelings, either positive or negative, about performing a specific behaviour (Fishbein \& Ajzen, 1980). It refers to a person's decision to perform a specific behaviour based on his/her estimation of the costs and benefits derived from the behaviour (Cheng, Lam \& Hsu, 2006). Previous research has established that consumers' attitude towards a behaviour influences their behavioural intention (Ahn \& Back, 2018; Bae \& Chang, 2021; Yusuf \& Busalim, 2018; Zhang et al., 2018). This finding is supported by the TPB, which states that a positive attitude leads to stronger behavioural intention (Fishbein \& Ajzen, 1980). Due to the growing population of Millennials and their proclivity for using technology and the internet (Ladhari, Gonthier \& Lajante, 2019), the current study focused on the effect of Millennials' attitude towards sharing OVAs on the intention to share OVAs. Based on extant findings, it was hypothesised that:

\section{H1: Attitude towards sharing OVAs positively influences the intention to share OVAs.}

\subsection{Perceived Entertainment Value, Perceived Informative Value, and Perceived Credibility Value}

Martins et al. (2019) defined perceived entertainment as the condition under which viewers have pleasurable, enjoyable, and fun feelings when watching advertisements. Gaber, Wright and Kooli (2019) showed that perceived entertainment has a positive influence on consumers' attitudes, as entertaining advertising attracts consumers' attention by stimulating feelings of joy and pleasure (Fishbein \& Ajzen, 1980). The relationship between perceived entertainment value and attitude towards behaviour has been tested in regard to online advertising as well, as Yang et al. (2017) revealed that entertainment value has a positive and significant relationship with the attitude towards internet advertising. However, limited studies have examined the effect of perceived entertainment value on the attitude towards sharing OVAs in particular. Based on extant findings, perceived entertainment value may positively influence the attitude towards sharing OVAs among Millennials. Thus, the study proposed the following hypothesis:

\section{H2: Perceived entertainment value positively influences the attitude towards sharing OVAs.}

Perceived informative value is the ability of advertisements to inform consumers and develop consumers' understanding about a product (Martins et al., 2019). Gaber, Wright and Kooli (2019) found that perceived informative value has a significant positive impact on the attitude towards Instagram advertisements. Similarly, Yang et al. (2017) demonstrated that information (i.e., perceived informative value) has a positive influence on the attitude towards internet advertising. Lenhart et al. (2010), likewise, concurred that perceived informative value can build positive or negative consumer attitudes towards online advertising by transferring information to consumers. However, the link between perceived informative value and the attitude towards sharing OVAs, in particular, is not well-established in the literature. In line with evidence from previous studies, it is plausible that perceived informative value positively influences Millennials' attitude towards sharing OVAs. Thus, the study proposed the following hypothesis:

\section{H3: Perceived informative value positively influences the attitude towards sharing OVAs.}

Perceived credibility value refers to whether or not people trust the content of advertisements (Erkan \& Evans, 2016). Specifically, it involves consumers' perceptions of the truthfulness, reliability, and believability of advertisements. A consumer may not respond or pay attention to advertising if they do not think it is trustworthy (Ha \& Lam, 2017). Consistent with this notion, Yang et al. (2017) revealed a significant relationship between perceived credibility value and the attitude towards internet advertising. Gaber, Wright and Kooli (2019) also found that perceived credibility enhances the attitude towards Instagram advertisements, on the basis that consumers develop a positive attitude when they feel the content of an advertisement is truthful and reliable (Ajzen \& Fishbein, 1980). Other empirical studies have corroborated that perceived credibility value in advertising positively affects consumers' attitude, which can reduce the rate of consumers who do not trust advertising (Saputra \& Fachira, 2014). Nevertheless, little is known about the role of perceived credibility value in the attitude towards sharing OVAs. Therefore, based on previous findings, the study postulated the following hypothesis:

H4: Perceived credibility value positively influences the attitude towards sharing OVAs. 


\subsection{Subjective Norms in Sharing OVAs}

Subjective norms are defined as the perceived views of close people who are influential in a person's decision making, such as business partners, colleagues, relatives, close friends, and family members (Park, 2000). Generally, the stronger the subjective norms, the higher the behavioural intention (Lim \& Dubinsky, 2005). The study by Kashif, Zarkada and Ramayah (2018) found that subjective norms strongly influence the ethical intentions of customer service managers. The same findings were discovered by Bai, Wang and Gong (2019) with regard to consumers' intention to purchase organic food. Relevant to this study, Lee, Ham and Kim (2013) examined the relationship between subjective norms and the intention to share OVAs, discovering a positive relationship between the two in the context of university students. In contrast, the current study postulated that this relationship would exist in a different population, i.e., Millennials. Thus, the current study developed the following hypothesis:

H5: Subjective norms in sharing OVAs positively influence the intention to share OVAs.

\subsection{Social Influence}

Social influence signifies the influence of media messages (e.g., mass media reports, expert opinions in press, etc.) on behavioural decisions (De Vries, Gensler \& Leeflang, 2012; Gunther et al., 2006; Lee et al., 2017). According to the TPB, consumers will develop positive subjective norms when they are influenced by such media messages (Fishbein \& Ajzen, 1980). In fact, past studies have consistently stated that social influence has a direct effect on subjective norms in behavioural intention. For example, AlHaderi and Ahmed (2015) found that social influence or social characteristics have a positive relationship with subjective norms in the intention to use technology. Similarly, De Vries, Gensler and Leeflang (2012) and Lee et al. (2017) showed that social influence, through subjective norms, has a positive impact on the intention to accept or use a technology. Social influence posits that a person affected by online indicators (e.g., number of comments, views, likes, and shares) is more likely to hold positive subjective norms in sharing OVAs. Therefore, the current study measured social influence as an antecedent to subjective norms in sharing OVAs, on the basis that most people are encouraged to share advertisements upon seeing media messages. Thus, this study hypothesised that:

\section{H6: Social influence positively influences subjective norms in sharing OVAs.}

\subsection{Perceived Behavioural Control (PBC) over Sharing OVAs}

According to the TPB, PBC refers to an individual's perception of control over performing a particular behaviour due to sufficient or accessible resources. When such resources are not available, the individual's behavioural intention will decrease, regardless of the fact that he/she has a favourable attitude or strong subjective norms towards the intended behaviour (Han, Hsu \& Sheu, 2010). Kashif, Zarkada and Ramayah (2018) verified that PBC has a positive and significant influence on the ethical intentions of customer service managers. The findings of Zhang et al. (2018) also demonstrated that PBC is positively related to young consumers' purchase intention of green housing. Also in the green sector, Maichum, Parichatnon and Peng (2016) revealed that PBC has a significant positive influence on the purchase intention for green products. The impact of PBC was found to be significant in the context of senior citizens' behavioural intention towards physical activities as well (Wu, Li \& Sung, 2016). Considering these findings, the current study attempted to bridge the gap by measuring PBC's effect on the intention to share OVAs among Millennials, who perceptibly have full control over this decision. Therefore, the study proposed the following hypothesis:

\section{H7: PBC over sharing OVAs positively influences the intention to share OVAs.}

\subsection{Self-efficacy}

Self-efficacy is defined as the ability and skill of an individual in performing a certain behaviour (Pavlou \& Fygenson, 2006). The TPB incorporates the concept of self-efficacy belief in PBC within a more general framework of the links among beliefs, attitudes, intention, and behaviour (Ajzen, 1991). Lee and Lee (2001) determined that self-efficacy has a significant effect on PBC over mobile advertising. This was echoed by Ghasemaghaei and Hassanein (2015), who found that self-efficacy through PBC has a positive impact on persuasion processes in online shopping. Likewise, Eastin (2002) and O'Cass and Fenech (2003) showed that self-efficacy via $\mathrm{PBC}$ has a positive effect on user intention towards the acceptance of online shopping. Despite these findings, the examination of self-efficacy remains scarce in terms of the intention to share OVAs. Given the established significant role of self-efficacy in behavioural intention via PBC, the present study assessed self-efficacy as an antecedent to PBC over sharing OVAs among Millennials. Thus, the following hypothesis was developed:

H8: Self-efficacy positively influences PBC over sharing OVAs. 


\subsection{Company Reputation}

Boateng and Okoe (2015) defined company reputation as judgement from others involved with a company, including customers, stockholders, and employees. According to Goldberg and Hartwick (1990), a positive reputation directs potential customers to reflect on advertising messages more positively, triggering the organism component of the S-O-R model. Consequently, most prior studies have conceded that company reputation has a positive relationship with behavioural intention. Haery, Ghorbani and Zamani (2014) showed that company reputation has a significant impact on behavioural intention in Iran through the roles of customer trust, customer commitment, and customer recognition. Similarly, Gorondutse, Hilman and Nasidi (2014) affirmed the positive effect of company reputation on customers' loyalty (i.e., purchase intention) in the food and beverage (F\&B) industry in Nigeria, in line with the TPB. Nevertheless, there are few studies that have examined the implications of company reputation for the intention to share OVAs among Millennials, which was addressed in this study. It is reasonable that a company's reputation, based on its past actions, may have a significant influence on this cohort's intention to share OVAs. Thus, it was hypothesised that:

\section{H9: Company reputation positively influences the intention to share OVAs.}

\subsection{Brand Awareness}

Brand awareness is described as prospective customers' capability to identify and remember that a brand belongs to a certain product category (Aaker, 1991). Shehzad et al. (2014) showed that brand awareness has a significant positive effect on consumer intention in the buying behaviour of Pakistani university students. This finding was corroborated by Vahdati and Mousavi (2016) in their study on brand awareness and customer purchase intention in Iran. In the context of OVA sharing, however, little is known about the link between brand awareness and behavioural intention. Therefore, based on the S-O-R model, the current study predicted a significant relationship between brand awareness (stimulus) and the intention to share OVAs (organism) among Millennials. When people recognise certain products or services, they are more aware of advertisements about the product/service and are more likely to share the OVAs with others (response). Therefore, the study posited the following hypothesis:

\section{H10: Brand awareness positively influences the intention to share OVAs.}

\subsection{Celebrity Endorsement}

Celebrity endorsement is known as any individual recognised by the public who uses this credit to appear as an influencer in advertisements (Rachbini, 2018). There are three dimensions of celebrity endorsement, i.e., attractiveness, trustworthiness, and expertise (Priyankara et al., 2017). Attractiveness directly influences the success of a communication message because an attractive endorser can motivate customers to accept the information given by the attractive source (McGuire, 1969). Accordingly, Abbas et al. (2018) found that an attractive celebrity exerts a positive effect on the mobile phone purchase intention of male customers. Trustworthiness involve the characteristics of self-respect, believability, and honesty exhibited by the endorser and observed by the customers (Erdogan, 1999). When customers believe the source is trustworthy, they are more likely to believe information from the source, which is why marketers strive to choose endorsers with such characteristics to deliver advertising messages to their customers. Lastly, an endorser's expertise is his/her qualifications that inspire customers' confidence and encourage them to buy the product (Wang, Kao \& Ngamsiriudom, 2017). Past research by Al-Absy et al. (2020) indicated that expertise has a positive influence on the perception and even purchase intention of target consumers. Therefore, the current study investigated celebrity endorsement through these three dimensions. Based on the S-O-R model, when Millennial internet users are influenced by a celebrity who endorses an advertisement (stimulus), their behavioural intention to share OVAs (organism) would increase and they would be more likely to share OVAs (response). Thus, the study proposed the following hypothesis:

H11: Celebrity endorsement positively influences the intention to share OVAs.

\subsection{Intention to Share OVAs}

Ajzen (1991) defined intention as the motivational factor that influences behaviour, reflected by an individual's willingness to exert effort in planning and performing the behaviour. Therefore, the stronger the intention to engage in a behaviour, the more likely its performance. The positive relationship between behavioural intention and actual behaviour was confirmed by De Pelsmaeker et al. (2017) in the setting of filled chocolate consumption in Belgium and Hungary. Using the TPB, Hsu and Huang (2010) found strong support for the formation of tourist behavioural intention but could not integrate tourists' intention with their actual behaviour, implying that behavioural intention may not necessarily lead to actual behaviour. However, Yang et al. (2017) found that consumer purchase intention positively influences their shopping behaviour upon watching OVAs. 
Therefore, the inconsistent findings in past studies call for more research on the link between the intention to share OVAs and actual sharing behaviour, which this study conducted in the context of Millennials. Thus, adding the construct of actual behaviour to the TPB model, it was hypothesised that:

H12: The intention to share OVAs positively influences actual OVA sharing behaviour.

\subsection{Moderating Role of Perceived Intrusiveness}

Edwards, Li and Lee (2002) defined perceived intrusiveness as the cause of irritation and annoyance that makes consumers watch an advertisement with a negative perspective. They examined forced exposure and psychological reactance as the antecedent and consequence, respectively, of the perceived intrusiveness of pop-up advertisements. Their findings indicated that perceived intrusiveness has a positive relationship with irritation and advertisement avoidance. Hoogma (2015) also found that perceived intrusiveness has a negative moderating effect on the effect of email marketing on repeat purchase behaviour and brand attitude. However, few studies have looked into perceived intrusiveness as a moderator between subjective norms, PBC, and behavioural intention. Therefore, the current study measured perceived intrusiveness as a moderator between the TPB factors (internal factors) and the intention to share OVAs among Millennials.

Perceived intrusiveness was proposed to moderate only the TPB factors (internal factors) rather than the external factors because the TPB factors influence individual perceptions of a certain phenomenon, which in turn affects behavioural intention and actual behaviour (Ajzen, 1991). This means that only internal factors influence individual aspects of behavioural intention, whereas external factors act through multiple aspects, such as the environment and past experience. Under conditions where perceived intrusiveness is low, the relationship between the TPB factors and the intention to share OVAs would be stronger. This is because the level of irritation and annoyance toward OVAs is lower, indirectly influencing consumers to develop a greater intention to share OVAs. In contrast, when perceived intrusiveness is high, it weakens the relationship between the TPB factors and the intention to share OVAs. In this situation, perceived intrusiveness makes consumers feel irritated and hampers their intention to share OVAs. Therefore, based on the PRT and evidence that perceived intrusiveness moderates the effect of internal factors on behavioural intention, the following hypotheses were proposed:

H13a: Perceived intrusiveness moderates the relationship between the attitude towards sharing OVAs and the intention to share OVAs, whereby a high level of perceived intrusiveness weakens the relationship.

H13b: Perceived intrusiveness moderates the relationship between subjective norms in sharing OVAs and the intention to share OVAs, whereby a high level of perceived intrusiveness weakens the relationship.

H13c: Perceived intrusiveness moderates the relationship between PBC over sharing OVAs and the intention to share OVAs, whereby a high level of perceived intrusiveness weakens the relationship.

Figure 1 illustrates the study's complete framework.

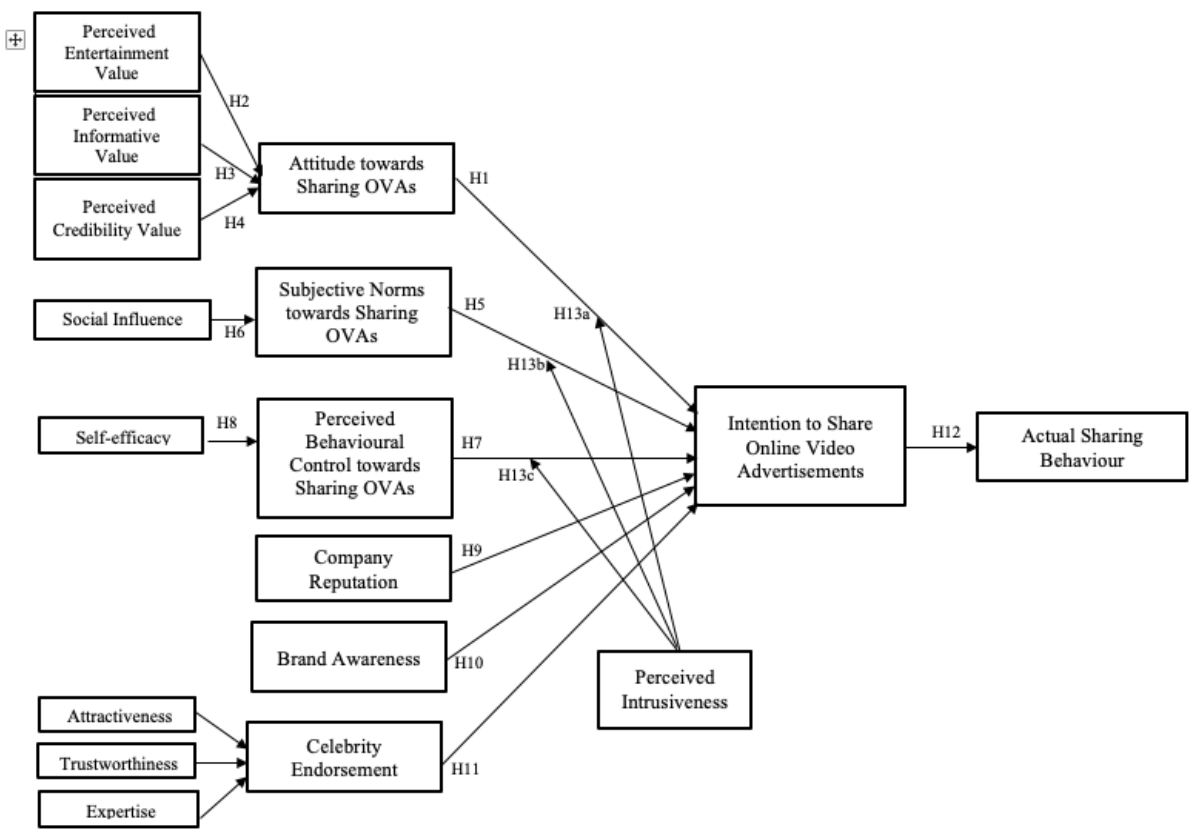

Figure 1. Research model 


\section{Methodology}

\subsection{Data Collection Method}

A self-administered questionnaire was designed for the purpose of data collection. To align with the main objective of this study (i.e., to examine Millennial consumers' actual OVA sharing behaviour), the questionnaire was distributed to respondents around shopping malls and universities in Selangor, Malaysia in online format using an iPad. The selection criteria were that respondents had to be: i) a Malaysian citizen currently staying in Selangor; ii) a Millennial aged between 19 and 38 years old; and iii) an owner of at least one social media account for the purpose of sharing OVAs. Of the 220 questionnaires distributed, 12 responses were eliminated due to high missing values. A total of 208 responses were finally included in subsequent analyses. This final sample size exhibited adequate statistical power, exceeding the minimum requirement of 189 samples prescribed by Faul et al.'s (2007) G*Power 3 software (parameters: $\mathrm{f}^{2}=0.15, \alpha=0.05$, and Power $=0.95$ ). Table 1 shows the demographic characteristics of the respondents.

Table 1. Demographic profile

\begin{tabular}{cccc}
\hline & Category & Frequency & Percent \\
\hline \multirow{2}{*}{ Gender } & Male & 82 & 39.40 \\
& Female & 126 & 60.60 \\
\hline \multirow{2}{*}{ Age } & $19-24$ years & 66.30 \\
& $25-30$ years & 26.40 & 7.20 \\
\hline \multirow{2}{*}{ Race } & $31-34$ years & 55 & 73.10 \\
& Malay & 15 & 19.70 \\
& Chinese & 152 & 4.80 \\
Occupation / Current Status & Indian & 41 & 2.40 \\
& Others & 10 & 3.80 \\
& Government Sector & 5 & 19.20 \\
& Private Sector & 8 & 2.40 \\
& Businessman & 40 & 1.40 \\
& Housewife & 5 & 71.20 \\
& Student & 3 & 1.90 \\
\hline \multirow{2}{*}{ Level of Education } & Unemployed & 148 & 2.40 \\
& Secondary & 4 & 6.30 \\
& Diploma & 5 & 76.40
\end{tabular}

\subsection{Measures}

Established scales were adapted from the literature for construct measurements. The measures of attitude towards sharing OVAs, subjective norms in sharing OVAs, and intention to share OVAs were adapted from Lee, Ham and Kim (2013). Perceived entertainment value, perceived credibility value, and perceived informative value were measured using the four-item scale developed by Martins et al. (2019), while social influence was measured using Lee et al.'s (2017) two-item scale. The items for PBC over sharing OVAs and self-efficacy were adapted from Pedersen (2005). The scales for company reputation (Boateng \& Okoe, 2015), brand awareness (Chow et al., 2017), and celebrity endorsement (Wang, Kao \& Ngamsiriudom, 2017) were adapted from previous studies as well. The moderator, perceived intrusiveness, was measured using a five-item scale from Chang, Rizal and Amin (2013). Lastly, to evaluate respondents' actual OVA sharing behaviour, this study adapted the measurement developed by Lagger, Lux and Marques (2012). The current study applied 5- and 7-point Likert scale to measure the constructs. All constructs were adapted, with slight modifications, from the literature (see Appendix A).

\section{Results}

\subsection{Common Method Variance (CMV)}

CMV analysis was conducted before other tests. As highlighted by Podsakoff et al. (2003), if the Harman's 
single factor test value is higher than $40.7 \%$, common method bias is believed to exist, especially in studies that measure consumer behaviour. The result showed that the highest variance explained by a single factor in this study was $36.52 \%$, which does not exceed $40.7 \%$. Thus, common method bias was not noteworthy in this study.

\subsection{Partial Least Squares Structural Equation Modelling (PLS-SEM)}

PLS-SEM was employed as the statistical tool to test the proposed hypotheses. As a variance-based technique, PLS-SEM was chosen in this study for several reasons. First, it is often used to maximise explained variance, which parallels the main objective of this study to examine the factors that drive Millennials' intention to share OVAs. Second, PLS-SEM has always performed better in prediction-oriented research, which is relevant to the present study (Hair et al., 2017). Third, this approach is capable of assessing a complex model with a large number of latent variables like that of this study, which comprised a higher-order construct (i.e., celebrity endorsement) and a moderating variable (i.e., perceived instructiveness) (Henseler, Ringle \& Sinkovics, 2009).

\subsection{Reflective Measurement Model Assessment}

The assessment of the reflective measurement model includes evaluating internal consistency reliability, convergent validity, and discriminant validity. First, as shown in Table 2, the composite reliability (CR) values for all constructs ranged from 0.876 to 0.972 (more than 0.70 ), indicating satisfactory internal consistency. Second, the value of average variance extracted (AVE) for all constructs were above the threshold of 0.50 (Fornell \& Larcker, 1981); thus, convergent validity was achieved.

Next, discriminant validity was measured based on the Heterotrait-Monotrait Ratio of Correlations (HTMT). As shown in Table 3, the HTMT values for all constructs were lower than 0.85 and passed the threshold limit of HTMT $_{.85}$ (Kline, 2011). Therefore, all the reflective constructs showed adequate discriminant validity.

Table 2. Reflective measurement model: Cronbach's Alpha, Factor loadings, CR, and AVE.

\begin{tabular}{|c|c|c|c|c|c|}
\hline Constructs & Items & Cronbach's Alpha & Loadings & AVE & $\mathrm{CR}$ \\
\hline \multirow{4}{*}{ Attitude toward sharing OVAs } & ATT 1 & 0.925 & 0.879 & 0.815 & 0.946 \\
\hline & ATT 2 & & 0.892 & & \\
\hline & ATT 3 & & 0.925 & & \\
\hline & ATT 4 & & 0.914 & & \\
\hline \multirow{4}{*}{ Perceived Entertainment Value } & ENT 1 & 0.962 & 0.945 & 0.897 & 0.972 \\
\hline & ENT 2 & & 0.946 & & \\
\hline & ENT 3 & & 0.955 & & \\
\hline & ENT 4 & & 0.941 & & \\
\hline \multirow{4}{*}{ Perceived Informative Value } & INF 1 & 0.922 & 0.899 & 0.810 & 0.945 \\
\hline & INF 2 & & 0.879 & & \\
\hline & INF 3 & & 0.920 & & \\
\hline & INF 4 & & 0.901 & & \\
\hline \multirow{4}{*}{$\begin{array}{c}\text { Perceived Credibility } \\
\text { Value }\end{array}$} & CRE 1 & 0.929 & 0.866 & 0.825 & 0.950 \\
\hline & CRE 2 & & 0.927 & & \\
\hline & CRE 3 & & 0.942 & & \\
\hline & CRE 4 & & 0.896 & & \\
\hline \multirow{4}{*}{ Subjective Norms } & SN 1 & 0.948 & 0.849 & 0.866 & 0.963 \\
\hline & SN 2 & & 0.950 & & \\
\hline & SN 3 & & 0.962 & & \\
\hline & $\mathrm{SN} 4$ & & 0.957 & & \\
\hline Social & SI 1 & 0.922 & 0.965 & 0.927 & 0.962 \\
\hline Influence & SI 2 & & 0.961 & & \\
\hline
\end{tabular}




\begin{tabular}{|c|c|c|c|c|c|}
\hline \multirow{3}{*}{ Perceived Behavioural Control } & PBC 1 & 0.869 & 0.882 & 0.792 & 0.920 \\
\hline & PBC 2 & & 0.892 & & \\
\hline & PBC 3 & & 0.897 & & \\
\hline \multirow{4}{*}{ Self-Efficacy } & SE 1 & 0.921 & 0.897 & 0.808 & 0.944 \\
\hline & SE 2 & & 0.889 & & \\
\hline & SE 3 & & 0.885 & & \\
\hline & SE 4 & & 0.924 & & \\
\hline \multirow{4}{*}{ Company Reputation } & CR 1 & 0.846 & 0.802 & 0.677 & 0.893 \\
\hline & CR 2 & & 0.890 & & \\
\hline & CR 3 & & 0.829 & & \\
\hline & $\mathrm{CR} 4$ & & 0.765 & & \\
\hline \multirow{4}{*}{$\begin{array}{c}\text { Brand } \\
\text { Awareness }\end{array}$} & BA 1 & 0.813 & 0.828 & 0.639 & 0.876 \\
\hline & BA 2 & & 0.850 & & \\
\hline & BA 3 & & 0.766 & & \\
\hline & BA 4 & & 0.750 & & \\
\hline \multirow{4}{*}{ Celebrity Endorsement -(Attractiveness) } & ATTR 1 & 0.850 & 0.807 & 0.689 & 0.899 \\
\hline & ATTR 2 & & 0.831 & & \\
\hline & ATTR 3 & & 0.844 & & \\
\hline & ATTR 4 & & 0.838 & & \\
\hline \multirow{4}{*}{ Celebrity Endorsement -(Trustworthiness) } & TRUST 1 & 0.840 & 0.768 & 0.675 & 0.893 \\
\hline & TRUST 2 & & 0.807 & & \\
\hline & TRUST 3 & & 0.845 & & \\
\hline & TRUST 4 & & 0.864 & & \\
\hline \multirow{4}{*}{ Celebrity Endorsement - (Expertise) } & EXP 1 & 0.833 & 0.768 & 0.668 & 0.889 \\
\hline & EXP 2 & & 0.859 & & \\
\hline & EXP 3 & & 0.813 & & \\
\hline & EXP 4 & & 0.826 & & \\
\hline \multirow{5}{*}{ Perceived Intrusiveness } & PI 1 & 0.947 & 0.854 & 0.821 & 0.958 \\
\hline & PI 2 & & 0.914 & & \\
\hline & PI 3 & & 0.932 & & \\
\hline & PI 4 & & 0.941 & & \\
\hline & PI 5 & & 0.887 & & \\
\hline \multirow{3}{*}{ Intention to share OVAs } & INT 1 & 0.960 & 0.969 & 0.926 & 0.974 \\
\hline & INT 2 & & 0.948 & & \\
\hline & INT 3 & & 0.970 & & \\
\hline Actual Sharing Behaviour & ASB1 & N/A & N/A & N/A & N/A \\
\hline
\end{tabular}

Note: Actual sharing behaviour was measured using a single item; thus, internal consistency and convergent validity values were not applicable. 
Table 3. Discriminant validity (HTMT).

\begin{tabular}{|c|c|c|c|c|c|c|c|c|c|c|c|c|c|c|c|c|}
\hline & ACTUAL & ATT & ATTR & BA & CR & EXP & INT & CRE & ENT & INF & PBC & PI & SE & SI & SN & TRUST \\
\hline \multicolumn{17}{|l|}{ ACTU } \\
\hline \multicolumn{17}{|l|}{$\mathbf{A L}$} \\
\hline \multirow[t]{2}{*}{ ATT } & 0.212 & & & & & & & & & & & & & & & \\
\hline & $\begin{array}{c}\text { CI.90 }(0.103, \\
0.318)\end{array}$ & & & & & & & & & & & & & & & \\
\hline \multirow[t]{2}{*}{ ATTR } & 0.200 & 0.529 & & & & & & & & & & & & & & \\
\hline & $\begin{array}{c}\text { CI.90 (0.098, } \\
0.317)\end{array}$ & $\begin{array}{c}\text { CI.90 }(0.422, \\
0.654)\end{array}$ & & & & & & & & & & & & & & \\
\hline \multirow[t]{2}{*}{ BA } & 0.209 & 0.517 & 0.529 & & & & & & & & & & & & & \\
\hline & $\begin{array}{c}\text { CI. } 90(0.107 \text {, } \\
0.337)\end{array}$ & $\begin{array}{c}\text { CI. } 90(0.408, \\
0.652)\end{array}$ & $\begin{array}{c}\text { CI.90 (0.396, } \\
0.656)\end{array}$ & & & & & & & & & & & & & \\
\hline \multirow[t]{2}{*}{ CR } & 0.135 & 0.531 & 0.510 & 0.624 & & & & & & & & & & & & \\
\hline & $\begin{array}{c}\text { CI. } 90(0.064, \\
0.242)\end{array}$ & $\begin{array}{c}\text { CI. } 90(0.432, \\
0.645)\end{array}$ & $\begin{array}{c}\text { CI.90 }(0.365 . \\
0.646)\end{array}$ & $\begin{array}{c}\text { CI. } 90(0.524, \\
0.716)\end{array}$ & & & & & & & & & & & & \\
\hline \multirow[t]{2}{*}{ EXP } & 0.184 & 0.356 & 0.540 & 0.533 & 0.523 & & & & & & & & & & & \\
\hline & $\begin{array}{c}\text { CI. } 90(0.087 \text {, } \\
0.292)\end{array}$ & $\begin{array}{c}\text { CI. } 90 \text { (0.237, } \\
0.497)\end{array}$ & $\begin{array}{c}\text { CI. } 90(0.385, \\
0.665)\end{array}$ & $\begin{array}{c}\text { CI. } 90(0.417, \\
0.664)\end{array}$ & $\begin{array}{c}\text { CI.90 (0.406, } \\
0.632)\end{array}$ & & & & & & & & & & & \\
\hline \multirow[t]{2}{*}{ INT } & 0,248 & 0.589 & 0.529 & 0.502 & 0.488 & 0.352 & & & & & & & & & & \\
\hline & $\begin{array}{c}\text { CI. } 90(0.132, \\
0.379)\end{array}$ & $\begin{array}{c}\text { CI. } 90(0.476, \\
0.687)\end{array}$ & $\begin{array}{c}\text { CI. } 90(0.425, \\
0.623)\end{array}$ & $\begin{array}{c}\text { CI. } 90(0.394, \\
0.600)\end{array}$ & $\begin{array}{c}\text { CI.90 (0.379, } \\
0.579)\end{array}$ & $\begin{array}{c}\text { CI. } 90(0.223, \\
0.480)\end{array}$ & & & & & & & & & & \\
\hline \multirow[t]{2}{*}{ CRE } & 0.182 & 0.693 & 0.480 & 0.519 & 0.588 & 0.289 & 0.464 & & & & & & & & & \\
\hline & $\begin{array}{c}\text { CI. } 90(0.070, \\
0.316)\end{array}$ & $\begin{array}{c}\text { CI. } 90 \text { (0.599, } \\
0.785)\end{array}$ & $\begin{array}{c}\text { CI. } 90(0.345, \\
0.614)\end{array}$ & $\begin{array}{c}\text { CI. } 90(0.400, \\
0.662)\end{array}$ & $\begin{array}{c}\text { CI.90 (0.449, } \\
0.707)\end{array}$ & $\begin{array}{c}\text { CI.90 (0.156, } \\
0.439)\end{array}$ & $\begin{array}{c}\text { CI. } 90(0.330, \\
0.586)\end{array}$ & & & & & & & & & \\
\hline \multirow[t]{2}{*}{ ENT } & 0.282 & 0.747 & 0.517 & 0.449 & 0.595 & 0.443 & 0.603 & 0.641 & & & & & & & & \\
\hline & $\begin{array}{c}\text { CI. } 90(0.184, \\
0.396)\end{array}$ & $\begin{array}{c}\text { CI. } 90(0.668, \\
0.810)\end{array}$ & $\begin{array}{c}\text { CI. } 90(0.404, \\
0.637)\end{array}$ & $\begin{array}{c}\text { CI. } 90(0.330, \\
0.564)\end{array}$ & $\begin{array}{c}\text { CI.90 (0.480, } \\
0.692)\end{array}$ & $\begin{array}{c}\text { CI. } 90(0.323, \\
0.576)\end{array}$ & $\begin{array}{c}\text { CI. } 90(0.495, \\
0.692)\end{array}$ & $\begin{array}{c}\text { CI. } 90(0.530, \\
0.746)\end{array}$ & & & & & & & & \\
\hline \multirow[t]{2}{*}{ INF } & 0.223 & 0.737 & 0.501 & 0.506 & 0.623 & 0.515 & 0.483 & 0.753 & 0.778 & & & & & & & \\
\hline & $\begin{array}{c}\text { CI. } 90(0.108, \\
0.348)\end{array}$ & $\begin{array}{c}\text { CI. } 90(0.654, \\
0.807)\end{array}$ & $\begin{array}{c}\text { CI. } 90(0.373, \\
0.628)\end{array}$ & $\begin{array}{c}\text { CI. } 90(0.370, \\
0.640)\end{array}$ & $\begin{array}{c}\text { CI.90 (0.498, } \\
0.736)\end{array}$ & $\begin{array}{c}\text { CI. } 90(0.379, \\
0.655)\end{array}$ & $\begin{array}{c}\text { CI. } 90(0.350, \\
0.591)\end{array}$ & $\begin{array}{c}\text { CI. } 90(0.678, \\
0.819)\end{array}$ & $\begin{array}{c}\text { CI. } 90(0.712, \\
0.830)\end{array}$ & & & & & & & \\
\hline \multirow[t]{2}{*}{ PBC } & 0.024 & 0.460 & 0.326 & 0.496 & 0.607 & 0.350 & 0.399 & 0.440 & 0.311 & 0.436 & & & & & & \\
\hline & $\begin{array}{c}\text { Cl. } 90(0.005, \\
0.025)\end{array}$ & $\begin{array}{c}\text { CI. } 90(0.342, \\
0.579)\end{array}$ & $\begin{array}{c}\text { CI. } 90(0.202, \\
0.445)\end{array}$ & $\begin{array}{c}\text { CI. } 90(0.387, \\
0.596)\end{array}$ & $\begin{array}{c}\text { CI.90 (0.494, } \\
0.700)\end{array}$ & $\begin{array}{c}\text { CI. } 90(0.208, \\
0.477)\end{array}$ & $\begin{array}{c}\text { CI. } 90(0.293, \\
0.501)\end{array}$ & $\begin{array}{c}\text { CI. } 90(0.312, \\
0.563)\end{array}$ & $\begin{array}{c}\text { CI. } 90(0.205, \\
0.439)\end{array}$ & $\begin{array}{c}\text { CI. } 90(0.301, \\
0.562)\end{array}$ & & & & & & \\
\hline \multirow[t]{2}{*}{ PI } & 0.045 & 0.106 & 0.104 & 0.089 & 0.078 & 0.177 & 0.193 & 0.089 & 0.158 & 0.071 & 0.092 & & & & & \\
\hline & $\begin{array}{c}\text { CI. } 90(0.013, \\
0.093)\end{array}$ & $\begin{array}{c}\text { CI. } 90 \text { (0.071, } \\
0.234)\end{array}$ & $\begin{array}{c}\text { C. } .90(0.088, \\
0.194)\end{array}$ & $\begin{array}{c}\text { CI. } 90(0.079, \\
0.198)\end{array}$ & $\begin{array}{c}\text { CI. } 90(0.068, \\
0.183)\end{array}$ & $\begin{array}{c}\text { CI. } 90(0.134, \\
0.310)\end{array}$ & $\begin{array}{c}\text { CI. } 90(0.081, \\
0.319)\end{array}$ & $\begin{array}{c}\text { CI. } 90(0.050, \\
0.215)\end{array}$ & $\begin{array}{c}\text { CI. } 90(0.077 \text {, } \\
0.288)\end{array}$ & $\begin{array}{c}\text { C. } .90(0.059, \\
0.197)\end{array}$ & $\begin{array}{c}\text { CI. } 90(0.076 \\
0.203)\end{array}$ & & & & & \\
\hline
\end{tabular}




\begin{tabular}{|c|c|c|c|c|c|c|c|c|c|c|c|c|c|c|c|}
\hline \multirow[t]{2}{*}{$\mathbf{S E}$} & 0.047 & 0.373 & 0.357 & 0.572 & 0.550 & 0.456 & 0.365 & 0.329 & 0.284 & 0.419 & 0.881 & 0.085 & & & \\
\hline & $\begin{array}{c}\text { CI.90 }(0.018, \\
0.070)\end{array}$ & $\begin{array}{c}\text { CI.90 }(0.265 \text {, } \\
0.501)\end{array}$ & $\begin{array}{c}\text { CI.90 (0.231, } \\
0.472)\end{array}$ & $\begin{array}{c}\text { CI.90 }(0.475 \text {, } \\
0.667)\end{array}$ & $\begin{array}{c}\text { CI.90 (0.436, } \\
0.647)\end{array}$ & $\begin{array}{c}\text { CI.90 (0.316, } \\
0.579)\end{array}$ & $\begin{array}{c}\text { CI.90 (0.238, } \\
0.473)\end{array}$ & $\begin{array}{c}\text { CI.90 (0.209, } \\
0.462)\end{array}$ & $\begin{array}{c}\text { CI.90 }(0.165 \text {, } \\
0.413)\end{array}$ & $\begin{array}{c}\text { CI.90 (0.295, } \\
0.552)\end{array}$ & $\begin{array}{c}\text { CI.90 }(0.832, \\
0.930)\end{array}$ & $\begin{array}{c}\text { CI.90 (0.065, } \\
0.186)\end{array}$ & & & \\
\hline \multirow[t]{2}{*}{ SI } & 0.280 & 0.675 & 0.627 & 0.550 & 0.572 & 0.476 & 0.585 & 0.531 & 0.655 & 0.579 & 0.471 & 0.083 & 0.412 & & \\
\hline & $\begin{array}{c}\text { CI.90 }(0.160, \\
0.391)\end{array}$ & $\begin{array}{c}\text { CI.90 (0.587, } \\
0.754)\end{array}$ & $\begin{array}{c}\text { CI.90 (0.531, } \\
0.716)\end{array}$ & $\begin{array}{c}\text { CI.90 (0.445, } \\
0.644)\end{array}$ & $\begin{array}{c}\text { CI.90 (0.472, } \\
0.658)\end{array}$ & $\begin{array}{c}\text { CI.90 }(0.348 \\
0.587)\end{array}$ & $\begin{array}{c}\text { CI.90 (0.486, } \\
0.663)\end{array}$ & $\begin{array}{c}\text { CI.90 (0.418, } \\
0.649)\end{array}$ & $\begin{array}{c}\text { CI.90 }(0.563, \\
0.736)\end{array}$ & $\begin{array}{c}\text { CI.90 (0.485, } \\
0.678)\end{array}$ & $\begin{array}{c}\text { CI.90 (0.363, } \\
0.575)\end{array}$ & $\begin{array}{c}\text { CI.90 (0.060, } \\
0.181)\end{array}$ & $\begin{array}{c}\text { CI.90 (0.295, } \\
0.530)\end{array}$ & & \\
\hline \multirow[t]{2}{*}{ SN } & 0.272 & 0.527 & 0.483 & 0.528 & 0.547 & 0.308 & 0.612 & 0.504 & 0.536 & 0.506 & 0.452 & 0.056 & 0.434 & 0.647 & \\
\hline & $\begin{array}{c}\text { CI.90 }(0.172, \\
0.394)\end{array}$ & $\begin{array}{c}\text { CI.90 (0.413, } \\
0.645)\end{array}$ & $\begin{array}{c}\text { CI.90 (0.359, } \\
0.602)\end{array}$ & $\begin{array}{c}\text { CI.90 (0.414, } \\
0.624)\end{array}$ & $\begin{array}{c}\text { CI. } 90(0.435, \\
0.641)\end{array}$ & $\begin{array}{c}\text { CI.90 (0.195, } \\
0.427)\end{array}$ & $\begin{array}{c}\text { CI.90 (0.522, } \\
0.692)\end{array}$ & $\begin{array}{c}\text { CI.90 }(0.390, \\
0.620)\end{array}$ & $\begin{array}{c}\text { CI.90 (0.431, } \\
0.635)\end{array}$ & $\begin{array}{c}\text { CI.90 (0.389, } \\
0.619)\end{array}$ & $\begin{array}{c}\text { CI.90 (0.334, } \\
0.567)\end{array}$ & $\begin{array}{c}\text { CI.90 (0.041, } \\
0.168)\end{array}$ & $\begin{array}{c}\text { CI.90 (0.307, } \\
0.549)\end{array}$ & $\begin{array}{c}\text { CI.90 }(0.560 \\
0.731)\end{array}$ & \\
\hline TRUS & 0.160 & 0.565 & 0.771 & 0.525 & 0.593 & 0.549 & 0.431 & 0.480 & 0.478 & 0.552 & 0.422 & 0.083 & 0.422 & 0.517 & 0.439 \\
\hline$T$ & $\begin{array}{c}\text { CI.90 (0.068, } \\
0.271)\end{array}$ & $\begin{array}{c}\text { CI.90 }(0.444, \\
0.683)\end{array}$ & $\begin{array}{c}\text { CI.90 (0.660, } \\
0.858)\end{array}$ & $\begin{array}{c}\text { CI.90 (0.386, } \\
0.656)\end{array}$ & $\begin{array}{c}\text { CI.90 (0.456, } \\
0.711)\end{array}$ & $\begin{array}{c}\text { CI.90 }(0.402, \\
0.710)\end{array}$ & $\begin{array}{c}\text { CI.90 (0.328, } \\
0.536)\end{array}$ & $\begin{array}{c}\text { CI.90 (0.340, } \\
0.624)\end{array}$ & $\begin{array}{c}\text { CI.90 }(0.358, \\
0.605)\end{array}$ & $\begin{array}{c}\text { CI.90 (0.411, } \\
0.694)\end{array}$ & $\begin{array}{c}\text { CI.90 }(0.288, \\
0.542)\end{array}$ & $\begin{array}{c}\text { CI.90 }(0.066, \\
0.204)\end{array}$ & $\begin{array}{c}\text { CI.90 (0.299, } \\
0.546)\end{array}$ & $\begin{array}{c}\text { CI.90 }(0.406, \\
0.621)\end{array}$ & $\begin{array}{c}\text { CI.90 }(0.307, \\
0.556)\end{array}$ \\
\hline
\end{tabular}

Note: ACTUAL (Actual sharing behaviour), ATT (Attitude toward sharing OVAs), ENT (Perceived entertainment value), INF (Perceived informative value), CRE (Perceived credibility value), SN (Subjective norms toward sharing OVAs), SI (Social influence), PBC (Perceived behavioural control over sharing OVAs), SE (Self-efficacy), CR

(Company reputation), BA (Brand awareness), CE (Celebrity Endorsement), ATTR (Celebrity endorsement-Attractiveness), TRUST (Celebrity endorsement-Trustworthiness),

EXP (Celebrity endorsement-Expertise), PI (Perceived Intrusiveness), INT (Intention to share OVAs) 


\subsection{Assessment of Higher-Order Construct (HOC)}

In this study, celebrity endorsement was conceptualised as Type II, that is, as a reflective-formative higher-order construct (HOC). In the first phase, the dimensions of celebrity endorsement (i.e., attractiveness, trustworthiness, and expertise) were evaluated as lower-order constructs in the reflective measurement model. In the second phase, the HOC construct was assessed using the formative measurement model, which includes tests for convergent validity, collinearity issues, and the dimensions' outer weight and significance (Hair et al., 2019; Ramayah et al., 2018).

First, a redundancy analysis of the celebrity endorsement construct was conducted by regressing all the dimensions to a global item. The convergent validity score for celebrity endorsement was 0.764 , exceeding the recommended threshold score of 0.70 (Hair et al., 2017). Thus, celebrity endorsement showed an adequate level of convergent validity. Next, the collinearity of the formative indicators was checked for all the dimensions of celebrity endorsements. As shown in Table 4, attractiveness (1.842), trustworthiness (1.861), and expertise (1.346) exhibited Variance Inflation Factor (VIF) scores below the threshold levels of 5.0 (Hair et al., 2017) and 3.3 (Diamantopoulos \& Siguaw, 2006), indicating that collinearity did not reach critical levels in this study. Lastly, the outer weights and significance of the dimensions were assessed using the bootstrapping approach. Expertise (outer weight $=0.948 ; \mathrm{t}$-value $=17.981)$ showed a significant impact on celebrity endorsement $(p<0.001)$, while attractiveness (outer weight $=0.084$, t-value $=0.969)$ and trustworthiness (outer weight $=0.022$, $\mathrm{t}$-value $=0.270)$ were insignificant $(\mathrm{p}<0.05)$. However, based on McCracken's (1989) suggestion, all the dimensions were retained to fully capture the content validity of celebrity endorsement.

Table 4. Results of Higher-order Construct - Celebrity Endorsement.

\begin{tabular}{ccccccc}
\hline \multirow{2}{*}{ Construct } & Sub-dimension & $\begin{array}{c}\text { Convergent } \\
\text { Validity }\end{array}$ & $\begin{array}{c}\text { Outer } \\
\text { Weight }\end{array}$ & $\begin{array}{c}\text { Outer } \\
\text { VIF }\end{array}$ & t-value & p-value \\
\hline \multirow{2}{*}{ Celebrity } & Attractiveness & & 0.084 & 1.842 & 0.969 & 0.333 \\
Endorsement & Trustworthiness & 0.764 & 0.022 & 1.861 & 0.270 & 0.787 \\
& Expertise & & 0.948 & 1.346 & $17.981^{* *}$ & $<0.001$ \\
\hline
\end{tabular}

\subsection{Structural Model Assessment}

The structural model includes assessments of lateral collinearity, path coefficients, coefficient of determination $\left(\mathrm{R}^{2}\right)$, effect size $\left(\mathrm{f}^{2}\right)$, and predictive relevance $\left(\mathrm{Q}^{2}\right)$. First, lateral collinearity measures the inner model for potential collinearity issues, where high correlations among the constructs indicate potential bias. The results indicated that VIF values ranged from 1.535 to 2.769 , which were below the threshold limit of 3.33 (Diamantopoulos \& Sigouw, 2006) and dispelled any concerns of collinearity. Then, the coefficient of determination $\left(\mathrm{R}^{2}\right)$ was evaluated to represent the degree of variance in the endogenous construct explained by all the exogenous constructs connected to it. Of the five endogenous constructs in the model, only one construct had weak explanatory power (i.e., actual sharing behaviour), whereas the other constructs had substantial explanatory power (from 0.368 to 0.624 ) (see Appendix B). Effect size was subsequently assessed to determine the extent to which an exogenous variable contributes to the endogenous variable's $R^{2}$ value (Hair et al., 2010). The results indicated that three constructs had a trivial effect size (PBC, CR, BA), six constructs had a small effect size (INF, CRE, ATT, SN, CE, INT), one construct had a medium effect size (ENT), and two constructs had a large effect size (SI, SE) (see Appendix C). Lastly, the present model displayed sufficient predictive relevance with a $\mathrm{Q}^{2}$ value more than zero (see Appendix D).

Then, path coefficients were assessed to evaluate the significance of the hypothesised relationships. A total of 12 direct hypotheses were developed, the results of which are summarised in Table 5. Attitude towards sharing OVAs $(\beta=0.262, p<0.05)$, subjective norms in sharing OVAs $(\beta=0.322, p<0.05)$, and celebrity endorsements $(\beta=0.143, p<0.05)$ were found to significantly influence the intention to share OVAs. However, PBC over sharing OVAs, company reputation, and brand awareness showed non-significant effects on the intention to share OVAs ( $\mathrm{p}>0.05)$.

Table 5. Results of hypothesis testing.

\begin{tabular}{lllll}
\hline Hypothesis & R/ship & Std. Beta & t-value & Decision \\
\hline H1 & ATT > INT & 0.262 & 3.130 & Supported \\
H2 & ENT > ATT & 0.402 & 6.155 & Supported \\
\hline
\end{tabular}




\begin{tabular}{lllll}
\hline H3 & INF $>$ ATT & 0.210 & 2.949 & Supported \\
H4 & CRE > ATT & 0.262 & 3.893 & Supported \\
H5 & SN > INT & 0.322 & 4.341 & Supported \\
H6 & SI > SN & 0.606 & 11.804 & Supported \\
H7 & PBC > INT & 0.019 & 0.291 & Unsupported \\
H8 & SE > PBC & 0.790 & 25.167 & Supported \\
H9 & CR > INT & 0.058 & 0.916 & Unsupported \\
H10 & BA > INT & 0.066 & 0.960 & Unsupported \\
H11 & CE > INT & 0.143 & 2.020 & Supported \\
H12 & INT > ASB & n/a & n/a & Supported \\
H13a & ATT*PI > INT & 0.109 & 2.080 & Supported \\
H13b & SN*PI > INT & 0.094 & 1.649 & Supported \\
H13c & PBC*PI > INT & 0.065 & 1.353 & Unsupported \\
\hline
\end{tabular}

Note: ASB (Actual sharing behaviour), ATT (Attitude toward sharing OVAs), ENT (Perceived entertainment value), INF (Perceived informative value), CRE (Perceived credibility value), SN (Subjective norms toward sharing OVAs), SI (Social influence), PBC (Perceived behavioural control over sharing OVAs), SE (Self-efficacy), CR (Company reputation), BA (Brand awareness), CE (Celebrity Endorsement), PI (Perceived Intrusiveness), INT (Intention to share OVAs)

\subsection{Moderation}

The moderation test returned two significant moderated relationships. This study followed the suggestion of Dawson (2014) by constructing interaction plots to better interpret the nature of the significant moderations (Fig. 2 and Fig. 3). First, the effect of attitude towards sharing OVAs on the intention to share OVAs is stronger when perceived intrusiveness is low $(\beta=0.109, \mathrm{p}<0.05)$. Likewise, the relationship between subjective norms in sharing OVAs and the intention to share OVAs is stronger when perceived intrusiveness is low $(\beta=0.094, \mathrm{p}<$ $0.05)$.

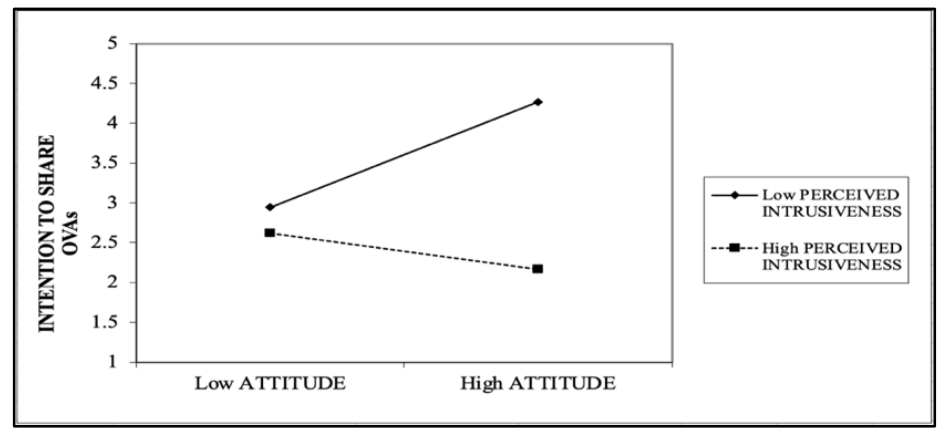

Figure 2. Moderating effect of perceived intrusiveness on the relationship between attitude towards sharing OVAs and intention to share OVAs

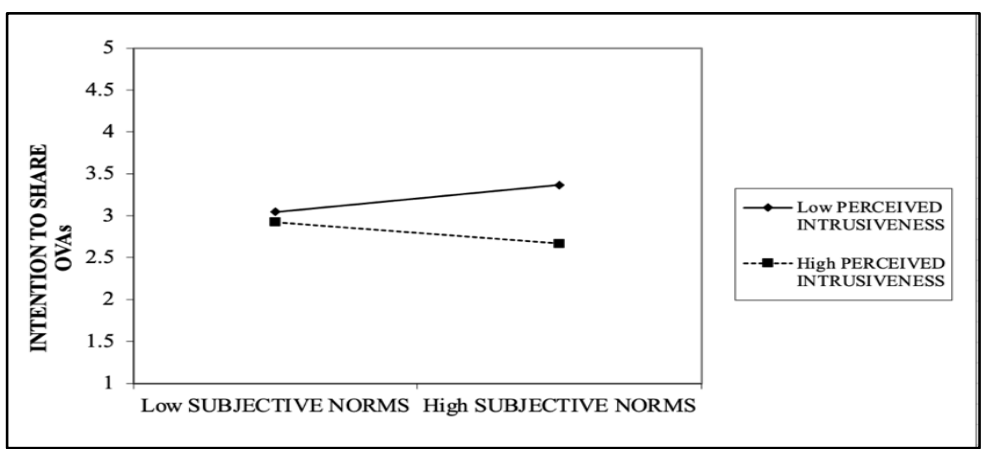

Figure 3. Moderating effect of perceived intrusiveness on the relationship between subjective norms in sharing OVAs and intention to share OVAs. 


\section{Discussion}

This study has revealed important internal, external, and moderating factors that influence Millennials' intention to share OVAs and actual OVA sharing behaviour. First, this study found that attitude towards sharing OVAs and subjective norms in sharing OVAs have positive effects on Millennials' intention to share OVAs. These findings suggest that when people have a positive attitude towards OVAs, they intend to share the OVAs with others. Similarly, Millennials are more likely to have positive intentions to share OVAs if the people surrounding them encourage and influence them to do so. These findings are in line with Lee, Ham and Kim (2013) discovery that attitude and subjective norms influence the intention to pass along OVAs. However, this study found that PBC toward the sharing of OVAs has an insignificant relationship with the intention to share OVAs. This finding is consistent with a past study by Jin and Kang (2011) and Lakmali and Kajendra (2017), which found a insignificant relationship between $\mathrm{PBC}$ and purchase intention. Although the Gen $\mathrm{Y}$ have the availability of resources to share, they might not do so if the OVAs do not appear interesting or important enough for them to share. Most of the time, OVAs that pop-up and appears to consumers are not related to them at all, and thus will not affect their intention to share.

Second, the findings reveal new antecedents to each TPB factor in the context of OVA sharing. Perceived entertainment value, informative value, and credibility value were found to significantly influence Millennials' attitude towards sharing OVAs. As mentioned by Ordun (2015), when viewers receive pleasant feelings of enjoyment (i.e., entertainment) from a certain OVA, they develop a positive attitude towards sharing the advertisement, which was confirmed by the studies of Yang et al. (2017) and Martins et al. (2019). In terms of perceived informative value, it is commonly believed that OVAs contain highly relevant and up-to-date information, which leads to a positive attitude towards sharing OVAs. Haida and Rahim (2015) and Yang et al. (2017) also indicated that information in advertisements positively affect the attitude of people watching OVAs. Additionally, the credibility of advertising positively impacts consumers' attitude as it reduces the level of distrust towards an advertisement (Saputra \& Fachira, 2014). This is in line with past research by Viswanathan and Jain (2013) that found a positive relationship between perceived credibility value and attitude toward advertising. Furthermore, social influence was shown to be a significant antecedent of subjective norms in sharing OVAs. As mentioned by Lee et al. (2017), social influence encompasses messages (i.e., mass media reports and expert opinions) spread by the media to manipulate individuals' behavioural decisions. Millennials are likely to be influenced by media messages on online platforms such as Facebook, Instagram, and YouTube (Viswanathan \& Jain, 2013). These platforms possess the ability to display the number of comments, likes, shares, and views received by an OVA, which are likely to influence this generation's perceptions. This finding is consistent with Bock et al.'s (2005) evidence that a person is more likely to have a positive or favourable feeling towards sharing intention when he/she is under the influence of their social network. Lastly, self-efficacy was shown to positively influence PBC over sharing OVAs. Hocevar, Flanagin and Metzger (2014), explained that people with a high level of self-efficacy tend to be more predictable and careful when observing product information to form an informed judgement about acquiring that product. However, as per the findings, PBC over sharing OVAs does not significantly influence Millennials' intention to share OVAs.

Third, this study found that celebrity endorsement, particularly by celebrities who are attractive, trustworthy, and knowledgeable, has a positive relationship with the intention to share OVAs among Millennials. According to Wang, Kao and Ngamsiriudom (2017) people are more likely to acknowledge information given by an attractive source. Additionally, Millennials are more likely to share OVAs when the celebrities that appear in advertisements are considered sincere, convincing, and dependable. When consumers have faith that the source is truthful, they perceive the communicated message to be highly credible (Erdogan, 1999). Finally, the element of expertise in celebrity endorsements plays an important role in influencing OVA sharing intention. Celebrity endorsements should be done by people who have expertise and qualifications, as this affects the level of persuasion and representation of a brand in advertising (Wang, Kao \& Ngamsiriudom, 2017). The finding of this study corroborates past research by $\mathrm{Han}$ and $\mathrm{Ki}$ (2010) and Priyankara et al. (2017), where celebrity endorsements with these three specific dimensions (attractiveness, trustworthiness, and expertise) were found to be significant in influencing behavioural intention. Conversely, this study found that company reputation and brand awareness have an insignificant relationship with the intention to share OVAs. It apparent that company reputation is important in the perception of OVAs, this may not necessarily lead to the sharing intention of OVAs. Besides, certain brands may be infamous for being excessively bad, hence, consumers may not want anything to do with them even though they are cognitively very aware of the brand.

Fourth, this study reveals perceived intrusiveness as moderator between the TPB factors and the intention to share OVAs. Only the effects of attitude and subjective norms on the intention to share OVAs are moderated by 
perceived intrusiveness. This indicates that when viewers perceive an OVA that has the element of intrusion or irritation, they shape a negative attitude towards it, which can influence their intention to share it. Similarly, if people are influenced by their family or friends who claim that OVAs are disturbing or annoying, it may negatively impact their intention to share OVAs. Meanwhile, PBC was not found to be moderated by perceived intrusiveness, which can be explained given the insignificant relationship of the construct with intention to share OVAs. Although the advertisements have the element of intrusive or not, it does not give any impact on intention to share OVAs, even though Gen Y have availability of resource to share.

Finally, as a complementary finding, the current study discovered that among the three types of OVA appeals (emotional, fear, and funny), emotional OVAs are the most likely to be shared by Millennials.

\subsection{Theoretical Implications}

In terms of theoretical implications, this study contributes to the literature in five ways. First, it extends the TPB framework by examining the intention to share OVAs and the actual sharing behaviour of OVAs among Millennials. However, based on findings of this study, it appears that only attitude and subjective norms are significant in predicting this cohort's intention to share OVAs, implying that the TRA possibly has superior explanatory power in explaining consumer behaviour in the context of OVAs. Second, this study investigated new antecedents to the TPB factors that influence Millennials' OVA sharing behaviour, which has rarely been examined in prior studies. The findings study prove that all the antecedents of the TPB factors are significant. These additions towards the TPB model bring a more holistic and complete view of the theory that better explains causal effects on consumer behaviour.

Third, underpinned by the S-O-R model, this study proposed three external factors (company reputation, brand awareness, celebrity endorsements) that influence Millennials' intention to share OVAs. Conversely, only celebrity endorsement was found to have a significant relationship with the intention to share OVAs. In this regard, the study adds to the extant literature by providing evidence on the importance of celebrity endorsements, which are within the control of organisation, for consumer behaviour in response to advertising. Fourth, this study incorporated a moderator to understand the magnitude of the TPB factors' effects on the intention to share OVAs. While previous research has explored ad avoidance and skipping behaviour in consumers' acceptance to advertising, the current study looked into perceived intrusiveness as a moderator of the relationship between Millennials' internal factors and their intention to share OVAs. The results of this study showed that attitude and subjective norms have significantly stronger positive effects on OVA sharing intention when perceived intrusiveness is low. Finally, this study investigated actual sharing behaviour and revealed that emotional OVA appeals are most likely to be shared by Millennials. This is a new contribution to advertising research, given that past studies have only measured behavioural intention rather than actual behaviour, especially in the context of OVA sharing based on specific ad appeals.

\subsection{Managerial Implications}

There are several important managerial implications that can be derived from this study. First, marketers should shift their marketing technique to online videos, as based on the findings, Millennials seem to watch online videos every day, proving that OVAs represent a strong tool to attract this group. Apart from that, the current study highlights that Millennials are more likely to share OVAs on entertainment. OVAs are thus a more suitable strategy for marketers who offer products or services under the entertainment category.

Second, to ensure that video advertisements on the online platform are received well, and to stimulate positive viewer attitudes towards sharing those ads, marketers should customise their marketing communication message by creating OVAs that contain elements of entertainment, information, and credibility. Third, seeing how social influence and subjective norms significantly influence the sharing of OVAs, marketers should concentrate on influencing customers who are already positively predisposed to the advertisements, so that these customers can spread and share the advertisements through their social network.

Moreover, marketers should strongly consider the use of celebrity endorsers in their video advertisements because celebrities with greater expertise in a particular field may have higher persuasive powers to influence viewers to watch and share OVAs. The study's findings also help distinguish that among the different ad types, emotional OVAs are most likely to be shared by Millennials due to emotional ads' ability to tug at one's heartstrings. Finally, the results of the study stress that low levels of perceived intrusiveness are imperative to strengthen the positive impacts of attitude and subjective norms on the intention to share OVAs. This means that it is critical for marketers to reduce viewers' perceptions of perceived intrusiveness in order to increase the likelihood of a brand's OVA being shared. Past studies indicated that ads are perceived to be more intrusive if they are poorly executed, being too long, played too often or having a style inconsistent with the containing 
website (Smith, 2011). Hence, marketers should strategize on embedding ads within videos that are highly relevant to those are sought after by viewers (for example, an ad on affordable Daiso products played during a video tutorial on managing finances) in order to reduce perceived intrusiveness.

\subsection{Limitations and Suggestions for Future Studies}

The main limitation of this study is its sample, which only included Millennials. Although the choice of sample is well-justified given the scope of the current study, it would be interesting to extend the study's research model to other generational cohorts, especially since all age groups in this day and age have access to the internet and are exposed to OVAs. Second, the current study took place solely in Selangor, Malaysia. Consequently, generalising the study's findings to other countries may not be entirely appropriate. Extending the study to other developing countries with high internet penetration rates, such as China and India, may bring forth different perspectives. Another concern is that OVAs can only be viewed by people who have internet access. Hence, the population of this study was limited to those who can access the internet and watch OVAs.

Next, although the current study identified the main determinants of OVA sharing behaviour, there is still room for future studies to explore other factors that may influence this phenomenon. For example, the impact of emotions on consumers in viral marketing (Botha \& Reyneke, 2013) or the moderating role of emotions may provide better explanatory power in future models. Other external factors should also be considered; for example, cause-related motivations for promoting trusted brands or other traits highly displayed by Millennials can be tested as antecedents to OVA sharing behaviour. Additionally, this study only focused on OVAs and did not measure other types of advertising that can be shared. To have a more holistic view of consumers' sharing behaviour in advertising, future research should look into other forms of shareable advertisements (i.e., print ads on internet platforms).

Furthermore, this study is limited by its self-report data, the weaknesses of which have been acknowledged in recent literature (Lucas, 2018; Beauchaine, Ben-David \& Sela, 2017). To overcome this weakness, it is recommended that future research engage in observational studies of behaviour or physiological movements (e.g., eye-tracking, pupilometer, facial muscle, sweat reaction, and brain activity) (Červenka et al., 2018). Additionally, future research can conduct follow-ups, such as via the physiology-measurement approach. Finally, this study employed a cross-sectional survey, where antecedents and outcomes were measured simultaneously; therefore, true causal inferences cannot be determined. Specifically, this study measured the actual sharing behaviour of OVAs at the point at which the survey was administered, which may have led to a slightly biased effect on actual behaviour. Finally, the study limited behavioural outcomes to three types of video ads (emotional, fear, and funny). Future studies should consider other types of ad appeals (e.g., rational ads) that people want to watch and share with others.

\section{Acknowledgement}

We would like to thank Geran Putra Malaysia, Universiti Putra Malaysia for funding our research. Grant number: 9677800

\section{References}

Aaker, D. A. (1991). Managing brand equity: Capitalizing on the Value of a Brand Name. New York, 28(1), 35-37.

Abbas, A., Afshan, G., Aslam, I., \& Ewaz, L. (2018). The effect of celebrity endorsement on customer purchase intention: A comparative study. Current Economics and Management Research, 4(1), 1-10.

Ahn, J., \& Back, K. J. (2018). Influence of brand relationship on customer attitude toward integrated resort brands: A cognitive, affective, and conative perspective. Journal of Travel \& Tourism Marketing, 35(4), 449-460. https://doi.org/10.1080/10548408.2017.1358239

Ajzen, I. (1991). The theory of planned behavior. Organizational behavior and human decision processes, 50(2), 179-211.

Al-Absy, M. S. M., Ismail, K. N. I. K., Chandren, S., \& Al-Dubai, S. A. A. (2020). Involvement of board chairmen in audit committees and earnings management: Evidence from Malaysia. The Journal of Asian Finance, Economics, and Business, 7(8), 233-246. https://doi.org/10.13106/jafeb.2020.vol7.no8.233

Alhaderi, S., \& Ahmed, F. (2015). The effect of social characteristic in the acceptance and continue usage for information technology in the public sector. International Journal of Business and Social Science, 6(7), 186-192.

Arif, I., Aslam, W., \& Siddiqui, H. (2020). Influence of brand related user-generated content through Facebook 
on consumer behaviour: A stimulus-organism-response framework. International Journal of Electronic Business, 15(2), 109-132. https://doi.org/10.1504/IJEB.2020.106502

Armitage, C. J., \& Conner, M. (2001). Efficacy of the theory of planned behaviour: A meta-analytic review. British journal of social psychology, 40(4), 471-499. https://doi.org/10.1348/014466601164939

Bae, S. Y., \& Chang, P. J. (2021). The effect of coronavirus disease-19 (COVID-19) risk perception on behavioural intention towards 'untact'tourism in South Korea during the first wave of the pandemic (March 2020). Current Issues in Tourism, 24(7), 1017-1035. https://doi.org/10.1080/13683500.2020.1798895

Bai, L., Wang, M., \& Gong, S. (2019). Understanding the antecedents of organic food purchases: The important roles of beliefs, subjective norms, and identity expressiveness. Sustainability, 11(11), 3045. https://doi.org/10.3390/su11113045

Baumeister, R. F., Catanese, K. R., \& Wallace, H. M. (2002). Conquest by force: A narcissistic reactance theory of rape and sexual coercion. Review of general psychology, 6(1), 92-135. https://doi.org/10.1037\%2F1089-2680.6.1.92

Beauchaine, T. P., Ben-David, I., \& Sela, A. (2017). Attention-deficit/hyperactivity disorder, delay discounting, and risky financial behaviors: A preliminary analysis of self-report data. PloS one, 12(5), e0176933. https://doi.org/10.1371/journal.pone.0176933

Belanche, D., Flavián, C., \& Pérez-Rueda, A. (2017). Understanding interactive online advertising: Congruence and product involvement in highly and lowly arousing, skippable video ads. Journal of Interactive Marketing, 37, 75-88. https://doi.org/10.1016/j.intmar.2016.06.004

Boateng, H., \& Okoe, A. F. (2015). Consumers' attitude towards social media advertising and their behavioural response. Journal of Research in Interactive Marketing. https://doi.org/10.1108/JRIM-01-2015-0012

Bock, G. W., Zmud, R. W., Kim, Y. G., \& Lee, J. N. (2005). Behavioral intention formation in knowledge sharing: Examining the roles of extrinsic motivators, social-psychological forces, and organizational climate. MIS quarterly, 87-111. https://doi.org/10.2307/25148669

Botha, E., \& Reyneke, M. (2013). To share or not to share: the role of content and emotion in viral marketing. Journal of Public Affairs, 13(2), 160-171. https://doi.org/10.1002/pa.1471

Brehm, J. W. (1996). A theory of psychological reactance. San Diego, CA: Academic Press.

Červenka, P., Naščáková, J., Bednárová, L., Daneshjo, N., \& Dudáš-Pajerská, E. (2018). The New Directions of Research in the Application of Effective Marketing Communication. In 2018 16th International Conference on Emerging eLearning Technologies and Applications (ICETA) (pp. 93-98). IEEE.

Chang, H. H., Rizal, H., \& Amin, H. (2013). The determinants of consumer behavior towards email advertisement. Internet Research. https://doi.org/10.1108/10662241311331754

Chen, C. C., \& Yao, J. Y. (2018). What drives impulse buying behaviors in a mobile auction? The perspective of the Stimulus-Organism-Response model. Telematics and Informatics, 35(5), 1249-1262. https://doi.org/10.1016/j.tele.2018.02.007

Cheng, S., Lam, T., \& Hsu, C. H. (2006). Negative word-of-mouth communication intention: An application of the theory of planned behavior. Journal of Hospitality \& Tourism Research, 30(1), 95-116. https://doi.org/10.1177\%2F1096348005284269

Chow, H. W., Ling, G. J., Yen, I. Y., \& Hwang, K. P. (2017). Building brand equity through industrial tourism. Asia Pacific Management Review, 22(2), 70-79. https://doi.org/10.1016/j.apmrv.2016.09.001

Connelly, L. M. (2008). Pilot studies. Medsurg Nursing, 17(6), 411.

Dawson, J. F. (2014). Moderation in management research: What, why, when, and how. Journal of business and psychology, 29(1), 1-19.

De Pelsmaeker, S., Schouteten, J. J., Gellynck, X., Delbaere, C., De Clercq, N., Hegyi, A., \& Dewettinck, K. (2017). Do anticipated emotions influence behavioural intention and behaviour to consume filled chocolates?. British Food Journal. https://doi.org/10.1108/BFJ-01-2016-0006

De Vries, L., Gensler, S., \& Leeflang, P. S. (2012). Popularity of brand posts on brand fan pages: An investigation of the effects of social media marketing. Journal of interactive marketing, 26(2), 83-91. https://doi.org/10.1016/j.intmar.2012.01.003

Diamantopoulos, A., \& Siguaw, J. A. (2006). Formative versus reflective indicators in organizational measure 
development: A comparison and empirical illustration. British journal of management, 17(4), $263-282$. https://doi.org/10.1111/j.1467-8551.2006.00500.x

Eastin, M. S. (2002). Diffusion of e-commerce: an analysis of the adoption of four e-commerce activities. Telematics and informatics, 19(3), 251-267. https://doi.org/10.1016/S0736-5853(01)00005-3

Edwards, S. M., Li, H., \& Lee, J. H. (2002). Forced exposure and psychological reactance: Antecedents and consequences of the perceived intrusiveness of pop-up ads. Journal of advertising, 31(3), 83-95. https://doi.org/10.1080/00913367.2002.10673678

eMarketers. (2016). Worldwide Ad Spending Growth Revised Downward. Retrieved March 24, 2019, from https://www.emarketer.com/Report/Worldwide-Ad-Spending-eMarketers-Updated-Estimates-Forecast-2015 2020/2001916

Erdogan, B. Z. (1999). Celebrity endorsement: A literature review. Journal of marketing management, 15(4), 291-314. https://doi.org/10.1362/026725799784870379

Erkan, I., \& Evans, C. (2016). The influence of eWOM in social media on consumers' purchase intentions: An extended approach to information adoption. Computers in Human Behavior, 61, 47-55. https://doi.org/10.1016/j.chb.2016.03.003

Fachryto, T., \& Achyar, A. (2018). Effect of online behavioral advertising implementation on attitude toward ad and purchase intention in Indonesian E-marketplace. Sriwijaya international journal of dynamic Economics and business, 2(2), 123-138. https://doi.org/10.29259/sijdeb.v2i2.123-138

Faul, F., Erdfelder, E., Lang, A. G., \& Buchner, A. (2007). G* Power 3: A flexible statistical power analysis program for the social, behavioral, and biomedical sciences. Behavior research methods, 39(2), 175-191.

Fishbein, M., \& Ajzen, A. (1980). Understanding Attitudes and Predicting Social Behaviour. Preventive-Hall. Inc., Englewood Cliffs.

Fornell, C., \& Larcker, D. F. (1981). Evaluating structural equation models with unobservable variables and measurement error. Journal of marketing research, $18(1), \quad 39-50$. https://doi.org/10.1177\%2F002224378101800104

Gaber, H. R., Wright, L. T., \& Kooli, K. (2019). Consumer attitudes towards Instagram advertisements in Egypt: The role of the perceived advertising value and personalization. Cogent Business \& Management, 6(1), 1618431. https://doi.org/10.1080/23311975.2019.1618431

Ghasemaghaei, M., \& Hassanein, K. (2015). Impact of persuasion processes on consumer attitude towards online shopping: The moderating role of product involvement and consumer self-efficacy.

Goldberg, M. E., \& Hartwick, J. (1990). The effects of advertiser reputation and extremity of advertising claim on advertising effectiveness. Journal of Consumer Research, 17(2), 172-179. https://doi.org/10.1086/208547

Gorondutse, A. H., Hilman, H., \& Nasidi, M. (2014). Relationship between corporate reputation and customer loyalty on Nigerian food and beverages industry: PLS approach. International Journal of Management and Business Research, 4(2), 125-136.

Gunther, A. C., Bolt, D., Borzekowski, D. L., Liebhart, J. L., \& Dillard, J. P. (2006). Presumed influence on peer norms: How mass media indirectly affect adolescent smoking. Journal of Communication, 56(1), 52-68. https://doi.org/10.1111/j.1460-2466.2006.00002.x

Ha, N. M., \& Lam, N. H. (2017). The effects of celebrity endorsement on customer's attitude toward brand and purchase intention. International Journal of Economics and Finance, 9(1), 64-77.

Haery, F. A., Ghorbani, H., \& Zamani, B. (2014). Investigating the relationship between corporate reputation and customer behavioral intentions through roles of customer trust, customer commitment and customer recognition. International Journal of Academic Research in Business and Social Sciences, 4(4), 470-484.

Haida, A., \& Rahim, H. L. (2015). Social media advertising value: A study on consumer's perception. Technology, 1(1), 1-8.

Hair, J. F., Black, W. C., Babin, B. J., \& Anderson, R. E. (2010). Multivariate Data Analysis: A Global Perspective. Pearson Education.

Hair, J. F., Hult, G. T. M., Ringle, C., \& Sarstedt, M. (2017). A Primer on Partial Least Squares Structural Equation Modelling (PLS-SEM). Sage Publications. 
Hair, J. F., Risher, J. J., Sarstedt, M., \& Ringle, C. M. (2019). When to use and how to report the results of PLS-SEM. European business review. https://doi.org/10.1108/EBR-11-2018-0203

Hall, A., Towers, N., \& Shaw, D. R. (2017). Understanding how Millennial shoppers decide what to buy. International Journal of Retail \& Distribution Management. https://doi.org/10.1108/IJRDM-11-2016-0206

Han, E., \& Ki, E. J. (2010). Developing a measure of celebrity reputation. Public Relations Review, 36(2), 199-201. https://doi.org/10.1016/j.pubrev.2009.10.013

Han, H., Hsu, L. T. J., \& Sheu, C. (2010). Application of the theory of planned behavior to green hotel choice: Testing the effect of environmental friendly activities. Tourism management, 31(3), 325-334. https://doi.org/10.1016/j.tourman.2009.03.013

Hansen, T., Jensen, J. M., \& Solgaard, H. S. (2004). Predicting online grocery buying intention: a comparison of the theory of reasoned action and the theory of planned behavior. International Journal of Information Management, 24(6), 539-550. https://doi.org/10.1016/j.ijinfomgt.2004.08.004

Henseler, J., Ringle, C. M., \& Sinkovics, R. R. (2009). The use of partial least squares path modeling in international marketing. In New challenges to international marketing. Emerald Group Publishing Limited. https://doi.org/10.1108/S1474-7979(2009)0000020014

Hocevar, K. P., Flanagin, A. J., \& Metzger, M. J. (2014). Social media self-efficacy and information evaluation online. Computers in Human Behavior, 39, 254-262. https://doi.org/10.1016/j.chb.2014.07.020

Hoogma, R. (2015). The Effects of E-Mail Marketing on Brand Loyalty.

Hsu, C. H., \& Huang, S. (2010). Formation of tourist behavioral intention and actual behavior. In 20107 th International Conference on Service Systems and Service Management (pp. 1-6). IEEE.

Jin, B., \& Kang, J. H. (2011). Purchase intention of Chinese consumers toward a US apparel brand: A test of a composite behavior intention model. Journal of consumer marketing. https://doi.org/10.1108/07363761111127617

Kashif, M., Zarkada, A., \& Ramayah, T. (2018). The impact of attitude, subjective norms, and perceived behavioural control on managers' intentions to behave ethically. Total Quality Management \& Business Excellence, 29(5-6), 481-501. https://doi.org/10.1080/14783363.2016.1209970

Kline, R. B. (2011). Principles and practice of structural equation modeling. New York: Guilford Press.

Kraus, M. (2017). Comparing Generation X and Generation Y on their preferred emotional leadership style. Journal of Applied Leadership and Management, 5, 62-75.

Ladhari, R., Gonthier, J., \& Lajante, M. (2019). Generation Y and online fashion shopping: Orientations and profiles. Journal of retailing and Consumer Services, 48, 113-121. https://doi.org/10.1016/j.jretconser.2019.02.003

Lagger, C., Lux, M., \& Marques, O. (2017). What makes people watch online videos: An exploratory study. Computers in Entertainment (CIE), 15(2), 1-31. https://doi.org/10.1145/3034706

Lakmali, A. D., \& Kajendra, K. (2017). Understanding Sri Lankan consumers' luxury fashion purchase intention: Status orientation as a complement to theory of planned behavior. International Journal of Innovative Research and Advanced Studies, 4(5), 294-299.

Lee, J., \& Lee, M. (2011). Factors influencing the intention to watch online video advertising. Cyberpsychology, Behavior, and Social Networking, 14(10), 619-624. https://doi.org/10.1089/cyber.2009.0305

Lee, J., Ham, C. D., \& Kim, M. (2013). Why people pass along online video advertising: From the perspectives of the interpersonal communication motives scale and the theory of reasoned action. Journal of Interactive Advertising, 13(1), 1-13. https://doi.org/10.1080/15252019.2013.768048

Lee, J., Kim, M., Ham, C. D., \& Kim, S. (2017). Do you want me to watch this ad on social media: The effects of norms on online video ad watching. Journal of Marketing Communications, 23(5), 456-472. https://doi.org/10.1080/13527266.2016.1232303

Lee, S. H., Yoon, S. H., \& Kim, H. W. (2021). Prediction of Online Video Advertising Inventory Based on TV Programs: A Deep Learning Approach. IEEE Access, 9, 22516-22527. https://doi.org/10.1109/ACCESS.2021.3056115

Lenhart, A., Ling, R., Campbell, S., \& Purcell, K. (2010). Teens and mobile phones: Text messaging explodes as teens embrace it as the centerpiece of their communication strategies with friends. Pew Internet \& American 
Life Project.

Leung, L., \& Chen, C. (2017). Extending the theory of planned behavior: A study of lifestyles, contextual factors, mobile viewing habits, TV content interest, and intention to adopt mobile TV. Telematics and Informatics, 34(8), 1638-1649. https://doi.org/10.1016/j.tele.2017.07.010

Lim, H., \& Dubinsky, A. J. (2005). The Theory of Planned Behaviour in E-Commerce: Making a Case for Interdependencies between Salient Beliefs. Psychology and Marketing, 22(10), 833-855. https://doi.org/10.1002/mar.20086

Lister, M. (2018). Workstreams. 37 Staggering Video Marketing Statistics for 2018. Retrieved from https://www.wordstream.com.

Lucas, R. E. (2018). Revaluating the strengths and weaknesses of self-report measures of subjective well-being. Handbook of well-being. Salt Lake City, UT: DEF Publishers.

Maichum, K., Parichatnon, S., \& Peng, K. C. (2016). Application of the extended theory of planned behavior model to investigate purchase intention of green products among Thai consumers. Sustainability, 8(10), 1077. https://doi.org/10.3390/su8101077

Malaysian Communication \& Multimedia Commission. (2017). Department of Statistics Malaysia. Internet User in Malaysia by State. Retrieved from https://www.mcmc.gov.my.

Malaysian Communication \& Multimedia Commission. (2021). Internet User Survey 2020. Retrieved from https://www.mcmc.gov.my

Malhotra, G., Mishra, S., \& Saxena, G. (2021). Consumers' psychological reactance and ownership in in-game advertising. Marketing Intelligence \& Planning. https://doi.org/10.1108/MIP-09-2020-0419

Marketing Land. (2016). Percentage of Viewers who Skip Advertisements. Retrieved from: https://marketingland.com

Martins, J., Costa, C., Oliveira, T., Gonçalves, R., \& Branco, F. (2019). How smartphone advertising influences consumers' purchase intention. Journal of Business Research, 94, 378-387. https://doi.org/10.1016/j.jbusres.2017.12.047

McCracken, G. (1989). Who is the Celebrity Endorser? Cultural Foundations of the Endorsement Process. Journal of Consumer Research, 16, 310-321. https://doi.org/10.1086/209217

McCue, T. J. (2020). The State of Online Video for 2020. Forbes. 5 Feb 2020. Retrieved February 6, 2021 from www.forbes.com/sites/tjmccue/2020/02/05/looking-deep-into-the-state-of-online-video-for-2020/?sh=4d54 3b8f2eac

McGuire, W. (1969). The nature of attitudes and attitude change (Vol. 3). Reading: Addison-Wesley Pub. Co.

Mehrabian, A., \& Russell, J. A. (1974). An approach to environmental psychology. The MIT Press.

Nunnally, J. C., \& Bernstein, I. H. (1994). The Assessment of Reliability. Psychometric Theory, 3, 248-292.

O'cass, A., \& Fenech, T. (2003). Web retailing adoption: exploring the nature of internet users Web retailing behaviour. Journal of Retailing and Consumer services, 10(2), 81-94. https://doi.org/10.1016/S0969-6989(02)00004-8

Ordun, G. (2015). Millennial (Gen Y) consumer behavior their shopping preferences and perceptual maps associated with brand loyalty. Canadian Social Science, 11(4), 40-55. http://dx.doi.org/10.3968/\%25x

Park, H. S. (2000). Relationships among attitudes and subjective norms: Testing the theory of reasoned action across cultures. Communication Studies, 51(2), 162-175. https://doi.org/10.1080/10510970009388516

Pashkevich, M., Dorai-Raj, S., Kellar, M., \& Zigmond, D. (2012). Empowering online advertisements by empowering viewers with the right to choose: The relative effectiveness of skippable video advertisements on YouTube. Journal of advertising research, 52(4), 451-457. https://doi.org/10.2501/JAR-52-4-451-457

Pavlou, P. A., \& Fygenson, M. (2006). Understanding and predicting electronic commerce adoption: An extension of the theory of planned behavior. MIS quarterly, 115-143. https://doi.org/10.2307/25148720

Pedersen, P. E. (2005). Adoption of mobile Internet services: An exploratory study of mobile commerce early adopters. Journal of organizational computing and electronic commerce, 15(3), 203-222. https://doi.org/10.1207/s15327744joce1503_2

Podsakoff, P. M., MacKenzie, S. B., Lee, J. Y., \& Podsakoff, N. P. (2003). Common method biases in behavioral 
research: A critical review of the literature and recommended remedies. Journal of applied psychology, $88(5), 879$.

Priyankara, R., Weerasiri, S., Dissanayaka, R., \& Jinadasa, M. (2017). Celebrity endorsement and consumer buying intention with relation to the Television advertisement for perfumes. Management Studies, 5(2), 128-148.

Quesenberry, K. A., \& Coolsen, M. K. (2019). Drama goes viral: Effects of story development on shares and views of online advertising videos. Journal of Interactive Marketing, 48, 1-16. https://doi.org/10.1016/j.intmar.2019.05.001

Rachbini, W. (2018). The influence of celebrity endorsements on purchase intention (a study on Vivo V7). Journal of Business and Management (IOSR-JBM), 20(8), 59-66.

Ramayah, T., Cheah, J., Chuah, F., Ting, H., \& Memon, M. A. (2018). Partial least squares structural equation modeling (PLS-SEM) using SmartPLS 3.0.

Sanne, P. N., \& Wiese, M. (2018). The theory of planned behaviour and user engagement applied to Facebook advertising. South African Journal of Information Management, 20(1), 1-10. https://doi.org/10.4102/sajim.v20i1.915

Saputra, S., \& Fachira, I. (2014). Users' Attitude Toward Skippable Ads On YouTube Trueview In-Stream-An Empirical Study Among College Students in Bandung. Journal of Business and Management, 3(8), 850-859.

Shehzad, U., Ahmad, S., Iqbal, K., Nawaz, M., \& Usman, S. (2014). Influence of brand name on consumer choice \& decision. Journal of Business and Management, 16(6), 72-76.

Smith, K. T. (2011). Digital marketing strategies that Millennials find appealing, motivating, or just annoying. Journal of Strategic marketing, 19(6), 489-499. https://doi.org/10.1080/0965254X.2011.581383

Vahdati, H., \& Mousavi Nejad, S. H. (2016). Brand Personality Toward Customer Purchase Intention: The Intermediate Role of Electronic Word-Of-Mouth and Brand Equity. Asian Academy of Management Journal, 21(2).

Viswanathan, V., \& Jain, V. (2013). A dual-system approach to understanding "generation Y" decision making. Journal of consumer marketing. https://doi.org/10.1108/JCM-07-2013-0649

Wang, S. W., Kao, G. H. Y., \& Ngamsiriudom, W. (2017). Consumers' attitude of endorser credibility, brand and intention with respect to celebrity endorsement of the airline sector. Journal of Air Transport Management, 60, 10-17. https://doi.org/10.1016/j.jairtraman.2016.12.007

Wu, J. H., Li, S. H., \& Sung, W. Y. (2016). The study of perceived environment and its relation to senior citizen's physical activity behavior intention. Journal of Business Research, 69(6), 2259-2264. https://doi.org/10.1016/j.jbusres.2015.12.039

Yang, K. C., Huang, C. H., Yang, C., \& Yang, S. Y. (2017). Consumer attitudes toward online video advertisement: YouTube as a platform. Kybernetes, 46(5), 840-853. https://doi.org/10.1108/K-03-2016-0038

YouTube Press. (2021). YouTube Statistics. Retrieved February 8, 2021, from https://www.youtube.com/intl/en-GB/about/press/

Yusuf, A. S., \& Busalim, A. H. (2018). Influence of e-WOM engagement on consumer purchase intention in social commerce. Journal of Services Marketing. https://doi.org/10.1108/JSM-01-2017-0031

Zhang, L., Chen, L., Wu, Z., Zhang, S., \& Song, H. (2018). Investigating young consumers' purchasing intention of green housing in China. Sustainability, 10(4), 1044. https://doi.org/10.3390/su10041044

Zhu, B., Kowatthanakul, S., \& Satanasavapak, P. (2019). Generation Y consumer online repurchase intention in Bangkok: Based on Stimulus-Organism-Response (SOR) model. International Journal of Retail \& Distribution Management. https://doi.org/10.1108/IJRDM-04-2018-0071

Zhu, L., Li, H., Wang, F. K., He, W., \& Tian, Z. (2020). How online reviews affect purchase intention: a new model based on the stimulus-organism-response (SOR) framework. Aslib Journal of Information Management. https://doi.org/10.1108/AJIM-11-2019-0308 


\section{Appendix A}

\begin{tabular}{|c|c|c|c|}
\hline Constructs & Items & Measurement Items & References \\
\hline \multirow{4}{*}{$\begin{array}{c}\text { Attitude towards } \\
\text { sharing OVAs } \\
\text { (ATT) }\end{array}$} & ATT1 & For me to share online video ads is good. & \multirow{4}{*}{$\begin{array}{l}\text { Lee, Ham \& } \\
\text { Kim (2013) }\end{array}$} \\
\hline & ATT2 & For me to share online video ads is valuable. & \\
\hline & ATT3 & For me to share online video ads is pleasant. & \\
\hline & ATT4 & For me to share online video ads is interesting. & \\
\hline \multirow{4}{*}{$\begin{array}{c}\text { Perceived } \\
\text { Entertainment } \\
\text { Value } \\
\text { (ENT) }\end{array}$} & ENT1 & I feel that online video ad is interesting. & \multirow{4}{*}{$\begin{array}{l}\text { Martins et al. } \\
\text { (2019) }\end{array}$} \\
\hline & ENT2 & I feel that online video ad is enjoyable. & \\
\hline & ENT3 & I feel that online video ad is entertaining. & \\
\hline & ENT4 & I feel that online video ad is pleasing. & \\
\hline \multirow{4}{*}{$\begin{array}{c}\text { Perceived } \\
\text { Informative Value } \\
\text { (INF) }\end{array}$} & INF1 & Online video ad provides timely information on products or services. & \multirow{4}{*}{$\begin{array}{l}\text { Martins et al } \\
\text { (2019) }\end{array}$} \\
\hline & INF2 & Online video ad supplies relevant information on product or services. & \\
\hline & INF3 & Online video ad is a good source of information. & \\
\hline & INF4 & $\begin{array}{l}\text { Online video ad is a good source of up-to-date products or services } \\
\text { information. }\end{array}$ & \\
\hline \multirow{4}{*}{$\begin{array}{c}\text { Perceived } \\
\text { Credibility Value } \\
\text { (CRE) }\end{array}$} & CRE1 & I feel that online video ad is convincing. & \multirow{4}{*}{$\begin{array}{l}\text { Martins et al } \\
\text { (2019) }\end{array}$} \\
\hline & CRE2 & I feel that online video ad is believable. & \\
\hline & CRE3 & I feel that online video ad is credible. & \\
\hline & CRE4 & I believe that online video ad is a good reference for purchasing products. & \\
\hline \multirow{4}{*}{$\begin{array}{c}\text { Subjective norms } \\
\text { towards Sharing } \\
\text { OVAs } \\
(\mathrm{SN})\end{array}$} & SN1 & $\begin{array}{l}\text { Many people whose opinion I value would approve of my sharing online } \\
\text { video ads if the ads are meaningful. }\end{array}$ & \multirow{4}{*}{$\begin{array}{l}\text { Lee, Ham \& } \\
\text { Kim (2013). }\end{array}$} \\
\hline & $\mathrm{SN} 2$ & $\begin{array}{l}\text { Many of my classmate/colleagues think that I need to share online video } \\
\text { ads if the ads are meaningful. }\end{array}$ & \\
\hline & SN3 & $\begin{array}{l}\text { Many of my close friends think that I need to share online video ads if } \\
\text { the ads are meaningful. }\end{array}$ & \\
\hline & SN4 & $\begin{array}{l}\text { Many people who are important to me think I need to share online video } \\
\text { ads if the ads are meaningful. }\end{array}$ & \\
\hline \multirow[t]{2}{*}{$\begin{array}{l}\text { Social Influence } \\
\text { (SI) }\end{array}$} & SI1 & $\begin{array}{l}\text { The ratings (e.g., star rating) show me that I should share a particular } \\
\text { online video ad. }\end{array}$ & \multirow{2}{*}{$\begin{array}{l}\text { Lee et al. } \\
\text { (2017). }\end{array}$} \\
\hline & SI2 & $\begin{array}{l}\text { The number of views shows me that I should share a particular online } \\
\text { video ad. }\end{array}$ & \\
\hline \multirow{3}{*}{$\begin{array}{l}\text { PBC towards } \\
\text { Sharing OVAs } \\
\text { (PBC) }\end{array}$} & $\mathrm{PBC} 1$ & I feel free to share online video ads that I like. & \multirow{3}{*}{$\begin{array}{l}\text { Pedersen } \\
(2005)\end{array}$} \\
\hline & $\mathrm{PBC} 2$ & Sharing online video ads is entirely within my control. & \\
\hline & $\mathrm{PBC} 3$ & I have the necessary means and resources to share online video ads. & \\
\hline \multirow{4}{*}{$\begin{array}{l}\text { Self-Efficacy } \\
\quad(\text { SE) }\end{array}$} & SE1 & I am able to share online video ads without the help of others. & \multirow{4}{*}{$\begin{array}{l}\text { Pedersen } \\
(2005)\end{array}$} \\
\hline & SE2 & I have the necessary time to share online video ads that are useful to me. & \\
\hline & SE3 & I have the knowledge and skills required to share online video ads. & \\
\hline & SE4 & I am able to share online video ads reasonably well on my own. & \\
\hline \multirow{4}{*}{$\begin{array}{c}\text { Company } \\
\text { Reputation } \\
\text { (CR) }\end{array}$} & CR1 & $\begin{array}{l}\text { I believe in online video ads of companies that are concerned about their } \\
\text { customers. }\end{array}$ & \multirow{4}{*}{$\begin{array}{l}\text { Boateng \& } \\
\text { Okoe (2015) }\end{array}$} \\
\hline & CR2 & $\begin{array}{l}\text { I believe in online video ads of companies I recommend to my friends } \\
\text { and relatives. }\end{array}$ & \\
\hline & CR3 & I believe in online video ads of companies that can be generally trusted. & \\
\hline & CR4 & $\begin{array}{l}\text { I believe in online video ads of companies that I am satisfied with my } \\
\text { overall experience. }\end{array}$ & \\
\hline \multirow{4}{*}{$\begin{array}{l}\text { Brand Awareness } \\
\text { (BA) }\end{array}$} & BA1 & I know the brand of the online video ads that I share. & \multirow{4}{*}{$\begin{array}{l}\text { Chow et al. } \\
\text { (2017). }\end{array}$} \\
\hline & BA2 & I can easily identify the brand of the online video ads that I share. & \\
\hline & BA3 & I think of the brand of the online video ads that I share first. & \\
\hline & BA4 & I am more familiar with the brand of the online video ads that I share to & \\
\hline
\end{tabular}


others.

Celebrity ATTR1 I prefer to watch online video ads with physically attractive (beautiful,

Endorsement - $\quad$ elegant, classy) endorser.

Attractiveness ATTR2 I think that attractiveness is an important characteristic for a celebrity

(ATTR) endorser in an online video ad.

ATTR3 I feel that a physically attractive endorser in an online video ad

Wang, Kao \&

Ngamsiriudom influences my purchase intention towards a celebrity endorsed brand.

(2017).

ATTR4 I remember a brand in an online video ad that is being endorsed by attractive celebrity more

\begin{tabular}{|c|c|c|c|}
\hline \multirow{4}{*}{$\begin{array}{c}\text { Celebrity } \\
\text { Endorsement - } \\
\text { Trustworthiness } \\
\text { (TRUST) }\end{array}$} & $\begin{array}{c}\text { TRUST } \\
1\end{array}$ & $\begin{array}{l}\text { I think that the online video ads with a trustworthy (dependable, honest, } \\
\text { sincere, reliable) endorser receive less negative recalls. }\end{array}$ & \multirow{4}{*}{$\begin{array}{c}\text { Wang, Kao \& } \\
\text { Ngamsiriudom } \\
\text { (2017). }\end{array}$} \\
\hline & $\begin{array}{l}\text { TRUST } \\
2\end{array}$ & $\begin{array}{l}\text { I feel that online video ads with a trustworthy endorser push me to } \\
\text { remember that ad and the product that is being endorsed. }\end{array}$ & \\
\hline & $\begin{array}{l}\text { TURST } \\
3\end{array}$ & The celebrity endorser in an online video ad is a trustworthy person. & \\
\hline & $\begin{array}{l}\text { TRUST } \\
4\end{array}$ & $\begin{array}{l}\text { I think a brand that is being endorsed by a trustworthy celebrity in an } \\
\text { online video ad is more respectable and desirable. }\end{array}$ & \\
\hline \multirow{5}{*}{$\begin{array}{c}\text { Celebrity } \\
\text { Endorsement - } \\
\text { Expertise } \\
\text { (EXP) }\end{array}$} & EXP1 & I think online video ads with a celebrity endorser who has expertise & \multirow{5}{*}{$\begin{array}{c}\text { Wang, Kao \& } \\
\text { Ngamsiriudom } \\
\text { (2017). }\end{array}$} \\
\hline & & (skilled, qualified, knowledgeable, and experienced) is more respectable. & \\
\hline & EXP2 & I pay more attention to online video ads using an expert celebrity. & \\
\hline & EXP3 & $\begin{array}{l}\text { I will buy a product if the celebrity endorsing it in online video ads is an } \\
\text { expert. }\end{array}$ & \\
\hline & EXP4 & $\begin{array}{l}\text { I think a brand that is being endorsed by an expert celebrity in online } \\
\text { video ads is more trustable. }\end{array}$ & \\
\hline \multirow{5}{*}{$\begin{array}{c}\text { Perceived } \\
\text { Intrusiveness } \\
\text { (PI) }\end{array}$} & PI1 & Online video ads are interfering. & \multirow{5}{*}{$\begin{array}{l}\text { Chang, Rizal \& } \\
\text { Amin (2013) }\end{array}$} \\
\hline & PI2 & Online video ads are disturbing. & \\
\hline & PI3 & Online video ads are obtrusive. & \\
\hline & PI4 & Online video ads are intrusive. & \\
\hline & PI5 & I feel forced to watch online video ads. & \\
\hline \multirow{3}{*}{$\begin{array}{c}\text { Intention to Share } \\
\text { OVAs } \\
\text { (INT) }\end{array}$} & INT1 & I plan to share online video ads. & \multirow{3}{*}{$\begin{array}{l}\text { Lee, Ham \& } \\
\text { Kim (2013) }\end{array}$} \\
\hline & INT2 & I will make an effort to share online video ads. & \\
\hline & INT3 & I intend to share online video ads. & \\
\hline
\end{tabular}

\section{Appendix B}

\begin{tabular}{ccc}
\hline Endogenous Variable & Level of $\mathrm{R}^{2}$ Assessment & Explanatory Power \\
\hline Actual Sharing Behaviour & 0.059 & Weak \\
Intention to share OVAs & 0.494 & Substantial \\
Attitude toward sharing OVAs & 0.601 & Substantial \\
Subjective Norms toward sharing OVAs & 0.368 & Substantial \\
Perceived Behavioural Control toward sharing OVAs & 0.624 & Substantial \\
\hline
\end{tabular}

Note: $\mathrm{R}^{2}$ score (0.26 - substantial, 0.13 - moderate and 0.02 - weak)

\section{Appendix C}

\begin{tabular}{|c|c|c|c|c|c|c|c|c|c|c|}
\hline Construct & ATT & Effect Size & SN & Effect Size & PBC & Effect Size & INT & Effect Size & ACT & Effect Size \\
\hline ENT & 0.179 & M & & & & & & & & \\
\hline INF & 0.040 & S & & & & & & & & \\
\hline CRE & 0.086 & S & & & & & & & & \\
\hline
\end{tabular}




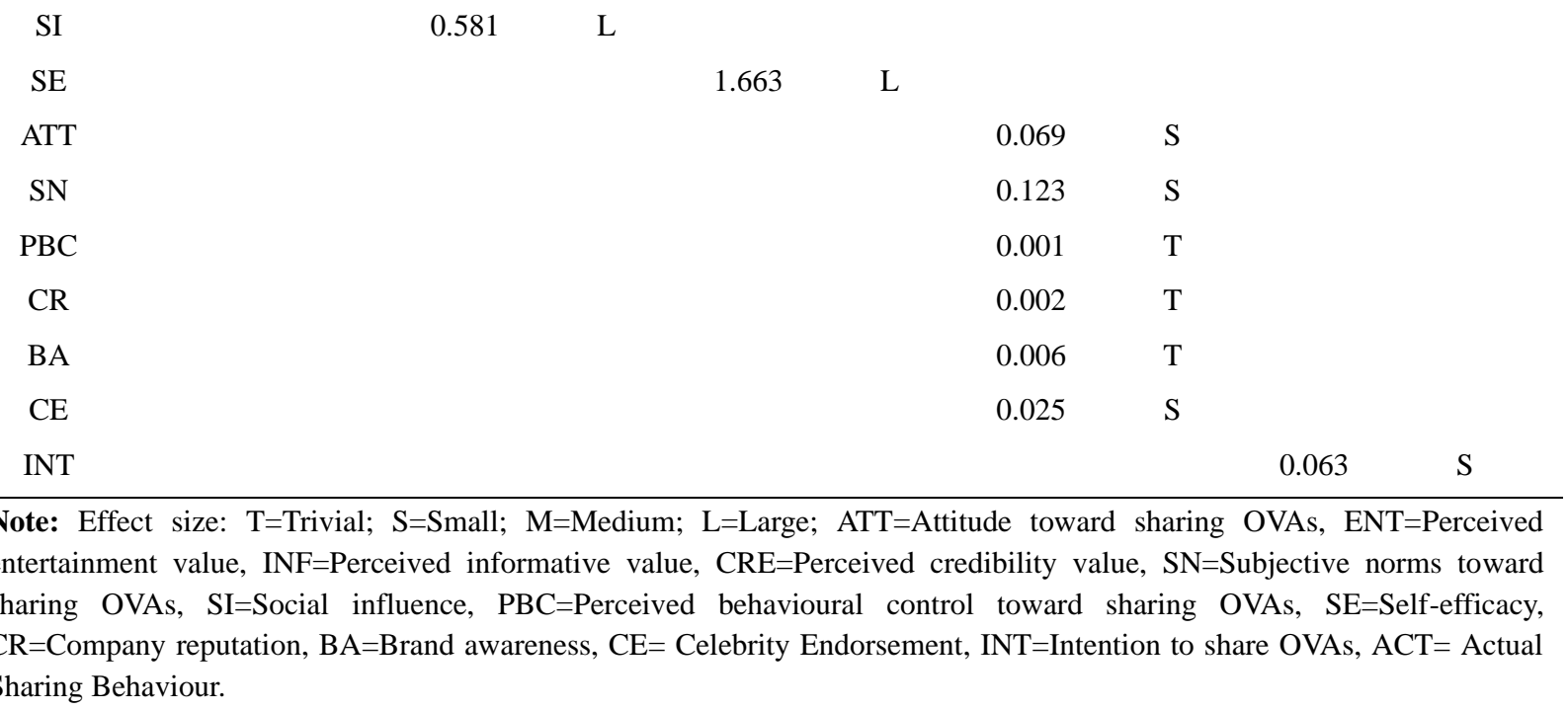

\section{Appendix D}

\begin{tabular}{cc}
\hline Construct & Predictive Relevance, $Q^{2}$ \\
\hline Actual Sharing Behaviour & 0.048 \\
Intention to share OVAs & 0.426 \\
Attitude toward sharing OVAs & 0.450 \\
Subjective Norms toward sharing OVAs & 0.297 \\
Perceived Behavioural Control toward sharing OVAs & 0.467 \\
\hline
\end{tabular}

Note: $Q^{2}>0$

\section{Copyrights}

Copyright for this article is retained by the author(s), with first publication rights granted to the journal.

This is an open-access article distributed under the terms and conditions of the Creative Commons Attribution license (http://creativecommons.org/licenses/by/4.0/). 